\title{
Design and Analysis of Multiscroll Chaotic Attractors From Saturated Function Series
}

\author{
Jinhu Lü, Member, IEEE, Guanrong Chen, Fellow, IEEE, Xinghuo Yu, Senior Member, IEEE, and \\ Henry Leung, Member, IEEE
}

\begin{abstract}
This paper initiates a saturated function series approach for chaos generation. The systematic saturated function series methodology developed here can create multiscroll chaotic attractors from a three-dimensional (3D) linear autonomous system with a simple saturated function series controller, including one-directional $n$-scroll, two-directional $n \times m$-grid scroll, and 3-D $n \times$ $m \times l$-grid scroll chaotic attractors. The dynamical behaviors and chaos generation mechanism of multiscroll systems are further investigated by analyzing the system trajectories. In particular, a two-dimensional (2D) Poincaré return map is rigorously derived for verifying the chaotic behaviors of the double-scroll chaotic attractor, which is a basic generator of various multiscroll chaotic attractors investigated in the paper.
\end{abstract}

Index Terms-Chaos generation, multiscroll chaotic attractor, saturated function series, saturated plateau.

\section{INTRODUCTION}

$\mathbf{I}$ T IS WELL KNOWN that chaos is useful and has great potential in many real-world engineering fields such as digital data encryption and secure communications, biomedical engineering, flow dynamics and liquid mixing, power-systems protection, and so on [1], [2].

Currently, generation of multiscroll chaotic attractors has been extensively studied and is no longer a very difficult task [3]-[17]. For example, Suykens et al. introduced several methods for generating $n$-scroll chaotic attractors using simple circuits [3]-[6], [9], [10], including the generalized Chua's circuit [4] and cellular neural network (CNN) [5], [7]. A piecewise-linear (PWL) implementation of $n$ double scrolls [3] was also proposed by Arena et al. in [7]. The essence of these methods is to add breakpoints in the PWL characteristic function of the nonlinear resistor in Chua's circuit [18], [19]. Tang et al. proposed a sine-function approach for creating $n$-scroll chaotic attractors, with a systematical circuit realization that can physically produce up to as many as ten scrolls visible on the oscilloscope [8],

Manuscript received February 16, 2004; revised May 27, 2004. This work was supported by the Hong Kong Research Grants Council under the CERG Grant CityU 1115/03E and the National Natural Science Foundation of People's Republic of China under Grant 60304017 and Grant 20336040/B06. This paper was recommended by Associate Editor Y. Nishio.

J. Lü is with the Institute of Systems Science, Academy of Mathematics and Systems Science, Chinese Academy of Sciences, Beijing 100080, China (e-mail: jhlu@iss.ac.cn).

G. Chen is with the Department of Electronic Engineering, City University of Hong Kong, Hong Kong, China (e-mail: gchen@ee.cityu.edu.hk).

$\mathrm{X}$. Yu is with the School of Electrical and Computer Engineering, RMIT University, Melbourne VIC 3001, Australia (e-mail: x.yu@rmit.edu.au).

H. Leung is with the Department of Electrical and Computer Engineering, University of Calgary, Calgary, AB T2N 1N4, Canada (e-mail: leungh@ ucalgary.ca).

Digital Object Identifier 10.1109/TCSI.2004.838151
[12]. Ozoguz et al. presented a nonlinear transconductor method for generating $n$-scroll attractors [11]. Lü et al. introduced a switching manifold approach for creating chaotic attractors with multiple-merged basins of attraction [14]. Yalcin et al. proposed a stair function method for generating one-directional (1-D) $n$-scroll, two-directional (2-D) $n \times m$-grid scroll, and three-directional (3-D) $n \times m \times l$-grid scroll chaotic attractors [10]. Hysteresis can also generate chaos [20]-[27]. Elwakil and Kennedy constructed a class of circuit-independent chaotic oscillators [20]. Elwakil et al. proposed some hysteresis chaotic oscillators [21]. Recently, Lü et al. presented a hysteresis series approach for creating 1-D $n$-scroll, 2-D $n \times m$-grid scroll, and 3-D $n \times m \times l$-grid scroll chaotic attractors [16]. Last but not least, Cafagna and Grassi [17] produced a ring Chua's circuit method for generating 1-D $n$-scroll and 2-D $n \times m$-grid scroll chaotic attractors. To this end, it should be noted that stair circuit, hysteresis circuit, and saturated circuit are the three kinds of basic circuits. Since it has been reported that stair circuit and hysteresis circuit can create 3-D multiscroll chaotic attractors [10], [15], [16], it is therefore very interesting to ask whether or not saturated circuit can also generate 3-D multiscroll chaotic attractors. This paper gives a positive answer to this question.

More precisely, this paper proposes a saturated function series approach for generating multiscroll chaotic attractors, including 1-D $n$-scroll, 2-D $n \times m$-grid scroll, and 3-D $n \times m \times l$-grid scroll chaotic attractors. It is noticed that the saturated function series is continuous, yet the stair function and hysteresis series are not continuous at all switching points. The dynamical behaviors and chaos generation mechanism of the multiscroll systems are further investigated by analyzing their trajectories. In particular, a 2-D Poincaré return map is rigorously derived for verifying the chaotic behaviors of the double-scroll chaotic attractor, which is a basic generator of various multiscroll chaotic attractors studied in the paper. In comparison, however, most of the reported multiscroll $(n \geq 3)$ chaotic attractors are only verified by using computer simulation and/or electronic circuits, where theoretical analysis and rigorous mathematical proofs are not available.

The rest of this paper is organized as follows. In Section II, the concept of saturated function series is introduced. The saturated function series approach is then introduced in Section III for creating multiscroll chaotic attractors, including 1-D $n$-scroll, 2-D $n \times m$-grid scroll, and 3-D $n \times m \times l$-grid scroll attractors. In Section IV, dynamical behaviors and chaos generation mechanism of the multiscroll systems are further investigated, and a 2D Poincaré return map is rigorously derived for verifying the chaotic behaviors of the double-scroll attractor. Conclusions are finally given in Section V. 


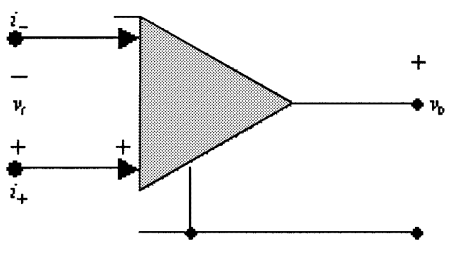

(a)

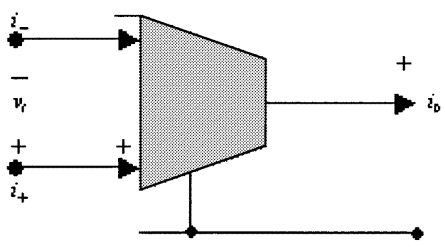

(b)

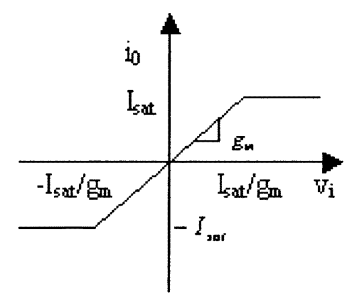

Fig. 1. (a) Opamp and its PWL model. (b) OTA and its PWL model.

\section{SATURATED FUnCTION SERIES}

This section introduces the saturated function series concept.

\section{A. Saturated Circuit}

It is well known that the saturated circuit is one of the basic PWL circuits. The PWL models for operational amplifiers (opamps) and operational transconductance amplifiers (OTAs) can be well characterized by saturated circuit [28]. From Fig. 1, one can see that the PWL approximations for opamps and OTAs are quite accurate [28]. It leads to a simple representation for opamp, which is in the linear region for $-E_{\text {sat }} \leq v_{0} \leq E_{\text {sat }}$, with voltage amplification $A_{v}$, positive saturation $E_{\text {sat }}$, and negative saturation $-E_{\mathrm{sat}}$, as follows:

$$
\left\{\begin{array}{l}
v_{0}=\frac{A_{v}}{2}\left(\left|v_{i}+\frac{E_{\mathrm{sat}}}{A_{v}}\right|-\left|v_{i}-\frac{E_{\mathrm{sat}}}{A_{v}}\right|\right) \\
i_{-}=i_{+}=0 .
\end{array}\right.
$$

It is called an opamp finite-gain model. In each of the three regions, the opamp can be characterized by a linear circuit.

Similarly, for the OTA, in the linear region $-I_{\text {sat }} \leq i_{0} \leq$ $I_{\text {sat }}$, with transconductance gain $g_{m}$, positive saturation $I_{\text {sat }}$, and negative saturation $-I_{\text {sat }}$, one has

$$
\left\{\begin{array}{l}
i_{0}=\frac{g_{m}}{2}\left(\left|v_{i}+\frac{I_{\mathrm{sat}}}{g_{m}}\right|-\left|v_{i}-\frac{I_{\mathrm{sat}}}{g_{m}}\right|\right) \\
i_{-}=i_{+}=0
\end{array}\right.
$$

\section{B. Saturated Function Series}

Consider the following saturated function:

$$
f_{0}(x ; k)= \begin{cases}k, & \text { if } x>1 \\ k x, & \text { if }|x| \leq 1 \\ -k, & \text { if } x<-1\end{cases}
$$

where $k>0$ is the slope of the middle segment, the upper radial $\left\{f_{0}(x ; k)=k \mid x \geq 1\right\}$ and the lower radial $\left\{f_{0}(x ; k)=\right.$ $-k \mid x \leq-1\}$ are called saturated plateaus, and the segment $\left\{f_{0}(x ; \bar{k})=k x|| x \mid \leq 1\right\}$ between the two saturated plateaus

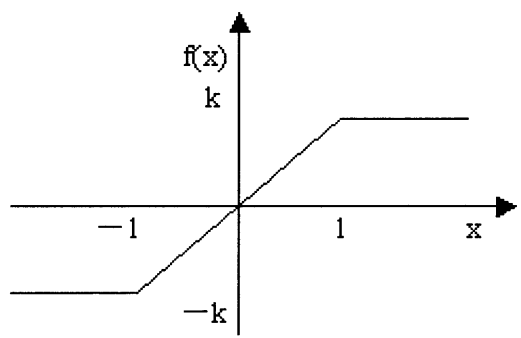

Fig. 2. Saturated function $f_{0}(x ; k)$.

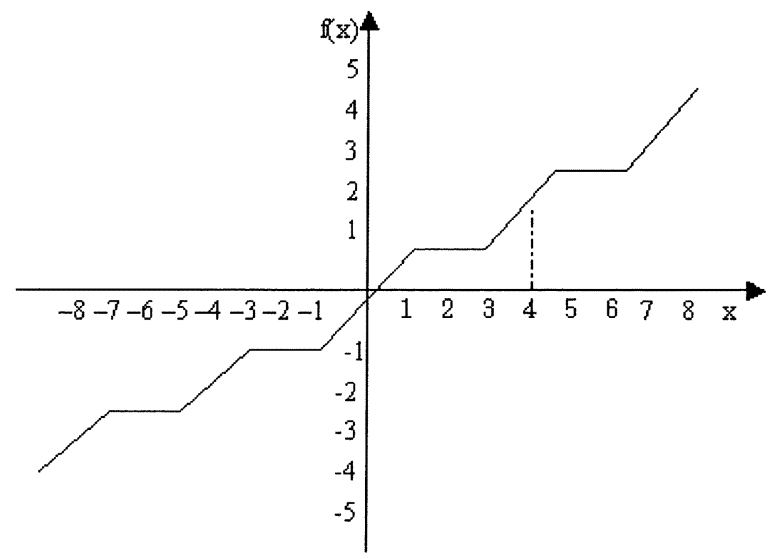

Fig. 3. Saturated function series with $k=1, h=4$.

is called the saturated slope. Fig. 2 shows the phase portrait of the saturated function $f_{0}(x ; k)$.

Definition 1: The following PWL function:

$$
f(x ; k, h, p, q)=\sum_{i=-p}^{q} f_{i}(x ; k, h)
$$

is called a saturated function series, where $k>0$ is the slope of saturated function series, $h>2$ is the saturated delay time of the saturated function series, $p$ and $q$ are positive integers, and

$$
f_{i}(x ; k, h)= \begin{cases}2 k, & \text { if } x>i h+1 \\ k(x-i h)+k, & \text { if }|x-i h| \leq 1 \\ 0, & \text { if } x<i h-1\end{cases}
$$

and

$$
f_{-i}(x ; k, h)= \begin{cases}0, & \text { if } x>-i h+1 \\ k(x+i h)-k, & \text { if }|x+i h| \leq 1 \\ -2 k, & \text { if } x<-i h-1\end{cases}
$$

One may recast the saturated function series $f(x ; k, h, p, q)$ as follows:

$$
\begin{aligned}
& f(x ; k, h, p, q) \\
& \quad= \begin{cases}(2 q+1) k, & \text { if } x>q h+1 \\
k(x-i h)+2 i k, & \text { if }|x-i h| \leq 1, \quad-p \leq i \leq q \\
(2 i+1) k, & \text { if } i h+1<x<(i+1) h-1 \\
-(2 p+1) k, & \text { if } x<-p h-1 .\end{cases}
\end{aligned}
$$

Fig. 3 shows the phase portrait of this saturated function series with $k=1, h=4$. It is noticed that saturated function series (5) is a PWL continuous function and has better analytical property such as the existence of solution and stability. However, one should notice that the stair function in [10] and the hysteresis series in [15], [16] are not continuous in all switching points. 
Consider the following 3-D linear autonomous system:

$$
\left\{\begin{array}{l}
\dot{x}=y \\
\dot{y}=z \\
\dot{z}=-a x-b y-c z
\end{array}\right.
$$

where $x, y, z$ are state variables, and $a, b, c$ are positive real constants. To guide the linear system (6) to generate chaotic behavior, it needs to add a nonlinear controller to stretch and fold the trajectories of the system repeatedly. Note that the PWL controller is the simplest nonlinear continuous controller. Here, the saturated function series (5) is used as the controller.

\section{Design of Multiscroll Chaotic AtTractors From SATURATEd FunCtion SERIES}

This section introduces a new and systematic method-saturated function series approach-for generating multiscroll chaotic attractors, including 1-D $n$-scroll, 2-D $n \times m$-grid scroll, and 3-D $n \times m \times l$-grid scroll chaotic attractors, from the linear autonomous system (6) by using a PWL controller.

Note that (6) has a unique equilibrium point $(0,0,0)$ and its corresponding characteristic equation is

$$
\lambda^{3}+c \lambda^{2}+b \lambda+a=0
$$

Denote $\hat{p}=b-(1 / 3) c^{2}, \hat{q}=(2 / 27) c^{3}-(1 / 3) b c+a$, and $\Delta=\left(a c^{3} / 27\right)-\left(b^{2} c^{2} / 108\right)-(a b c / 6)+\left(b^{3} / 27\right)+\left(a^{2} / 4\right)$. Solving (7) gives

and

$$
\lambda_{1}=-\frac{c}{3}+\sqrt[3]{-\frac{\hat{q}}{2}+\sqrt{\Delta}}+\sqrt[3]{-\frac{\hat{q}}{2}-\sqrt{\Delta}}
$$

$$
\begin{aligned}
\lambda_{2,3}= & -\frac{c}{3}-\frac{1}{2}\left(\sqrt[3]{-\frac{\hat{q}}{2}+\sqrt{\Delta}}+\sqrt[3]{-\frac{\hat{q}}{2}-\sqrt{\Delta}}\right) \\
& \pm \frac{\sqrt{3}}{2} i\left(\sqrt[3]{-\frac{\hat{q}}{2}+\sqrt{\Delta}}-\sqrt[3]{-\frac{\hat{q}}{2}-\sqrt{\Delta}}\right) \\
\equiv & \alpha \pm \beta i .
\end{aligned}
$$

Numerical computations show that the linear system (6) with a saturated function series controller will produce chaotic behavior under the conditions of $\lambda_{1}<0, \alpha>0$, and $\beta \neq 0$. That is, (7) has a negative eigenvalue and one pair of complex conjugate eigenvalues with positive real parts. Moreover, the equilibrium point $(0,0,0)$ is a two dimensionally unstable saddle, called a saddle point of index 2 [17]-[19].

In the following, assume that

$$
\begin{aligned}
& \Delta=\frac{a c^{3}}{27}-\frac{b^{2} c^{2}}{108}-\frac{a b c}{6}+\frac{b^{3}}{27}+\frac{a^{2}}{4}>0 \\
& \lambda_{1}=-\frac{c}{3}+\sqrt[3]{-\frac{\hat{q}}{2}+\sqrt{\Delta}}+\sqrt[3]{-\frac{\hat{q}}{2}-\sqrt{\Delta}}<0 \\
& \alpha=-\frac{c}{3}-\frac{1}{2}\left(\sqrt[3]{-\frac{\hat{q}}{2}+\sqrt{\Delta}}+\sqrt[3]{-\frac{\hat{q}}{2}-\sqrt{\Delta}}\right)>0 .
\end{aligned}
$$

\section{A. New Double Scroll Chaotic Attractor}

Here, the saturated function $f_{0}(x ; k)$ is chosen as a controller to guide system (6) to generate chaos. The controlled system is described by

$$
\left\{\begin{array}{l}
\dot{x}=y \\
\dot{y}=z \\
\dot{z}=-a x-b y-c z+d_{1} f_{0}(x ; k)
\end{array}\right.
$$

where $f_{0}(x ; k)$ is defined by ( 3$)$.

When $a=b=c=d_{1}=0.7, k=10$, system (11) has a double-scroll chaotic attractor as shown in Fig. 4(a). Fig. 4(b) shows the $x-y$ plane projection of the double-scroll attractor; Fig. 4(c) shows that the variable $x(t)$ spirals around two values, \pm 10 , making random excursions between these two values which correspond to the centers of the two scrolls in the attractor; Fig. 4(d) shows the Poincaré map on section $y=0$, where it is clear that the trajectories are folded and the corresponding double-scroll map reported in [9] can also be observed.

Obviously, system (11) has three equilibria, $S_{ \pm}( \pm 10,0,0)$ and $S_{0}(0,0,0)$, which correspond to the three PWL parts of the saturated function $f_{0}(x ; k)$ in Fig. 2 , respectively. Equilibria $S_{ \pm}$ possess eigenvalues $\lambda_{1}=-0.8480, \lambda_{2,3}=0.0740 \pm 0.9055 i$, which are called saddle points of index 2 since the two complex conjugate eigenvalues have positive real parts [17]-[19]. Equilibrium point $S_{0}$ has eigenvalues $\lambda_{1}=1.5309, \lambda_{2,3}=$ $-1.1154 \pm 1.6944 i$, which is called saddle point of index 1 since the real eigenvalue is positive [17]. It is noticed that the scrolls are generated only around the equilibria of saddle points of index 2 [17]-[19]. Moreover, equilibria $S_{ \pm}$correspond to the two saturated plateaus, which are responsible for generating the two scrolls in the double-scroll attractor. However, the equilibrium point $S_{0}$ corresponds to the saturated slope and is responsible for connecting these two symmetrical scrolls. The Lyapunov exponent spectrum and Lyapunov dimension can be calculated by using the numerical methods described in [29], yielding $\mathrm{LE}_{1}=0.1042, \mathrm{LE}_{2}=0, \mathrm{LE}_{3}=-0.8043$, and $\mathrm{LD}=2.1297$.

According to the above analysis, this new double-scroll attractor is similar to but different from Chua's double-scroll attractor [18] since Chua's double-scroll attractor is created by using Chua's circuit and the two circuits are not topologically equivalent.

\section{B. 1-D n-Scroll Chaotic Attractors}

In the following, to create $n$-scroll chaotic attractors $(n \geq$ 3 ), a saturated function series controller is added to system (6), leading to

$$
\left\{\begin{array}{l}
\dot{x}=y \\
\dot{y}=z \\
\dot{z}=-a x-b y-c z+d_{1} f\left(x ; k_{1}, h_{1}, p_{1}, q_{1}\right)
\end{array}\right.
$$

where $f\left(x ; k_{1}, h_{1}, p_{1}, q_{1}\right)$ is defined by (5), and $a, b, c, d_{1}$ are positive constants.

Assume that

$$
\begin{aligned}
d_{1} k_{1} & >a \\
2 d_{1} k_{1} & \geq a h_{1} \\
\max \left\{p_{1}, q_{1}\right\} \frac{\left|a h_{1}-2 k_{1} d_{1}\right|}{d_{1} k_{1}-a} & \leq 1 \\
\left(2 d_{1} k_{1}-a h_{1}\right)\left(q_{1}-1\right) & <a h_{1}-d_{1} k_{1}-a .
\end{aligned}
$$



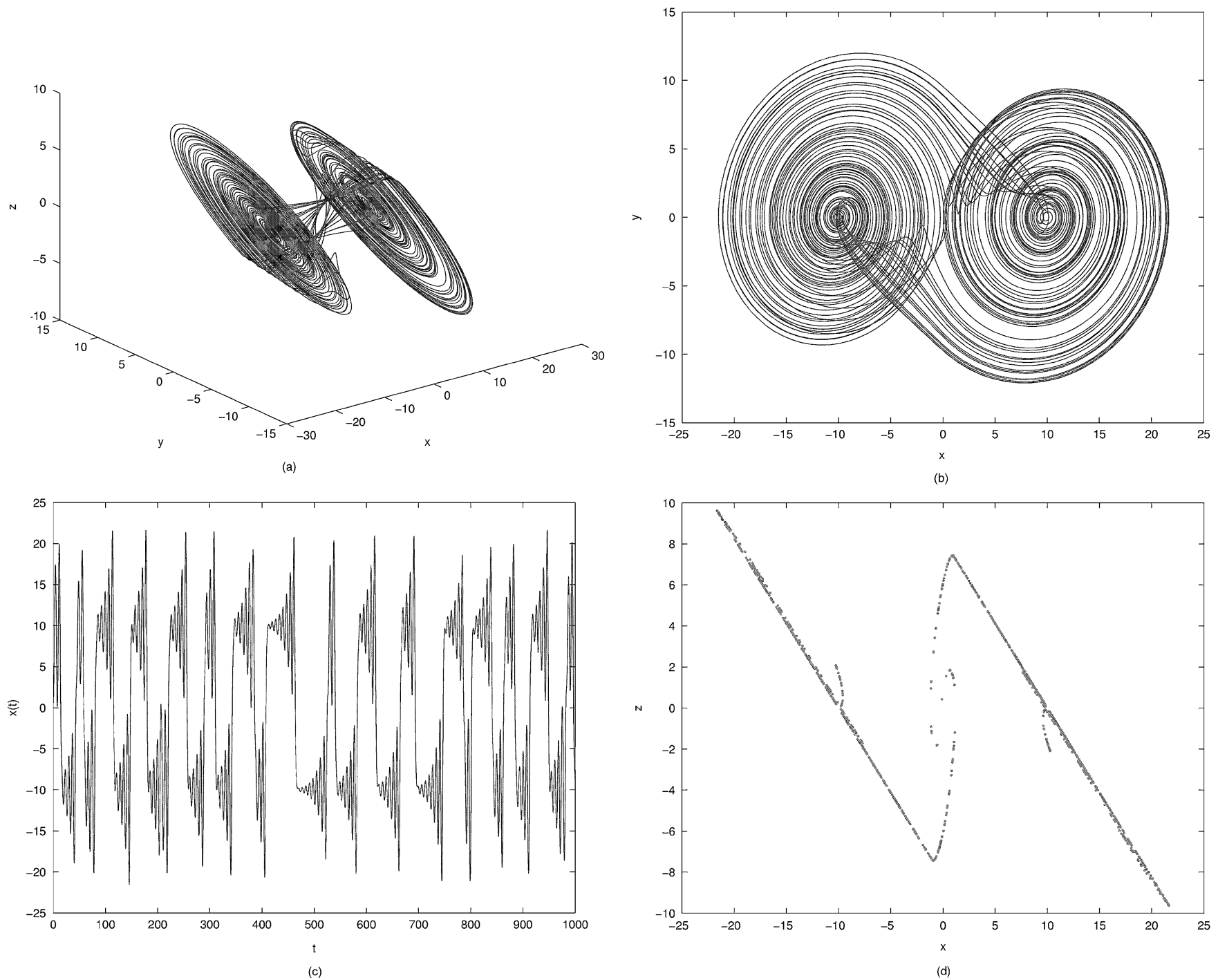

Fig. 4. Double-scroll chaotic attractor. (a) $x-y-z$ space. (b) $x-y$ plane projection. (c) Variable $x(t)$. (d) Poincaré map on section $y=0$.

Obviously, all $2\left(p_{1}+q_{1}\right)+3$ equilibrium points of system (12) are located along the $x$-axis, and can be classified into two different sets

$$
\begin{aligned}
A_{x}= & \left\{-\frac{\left(2 p_{1}+1\right) d_{1} k_{1}}{a}, \frac{\left(-2 p_{1}+1\right) d_{1} k_{1}}{a}, \ldots,\right. \\
& \left.\frac{\left(2 q_{1}+1\right) d_{1} k_{1}}{a}\right\}
\end{aligned}
$$

and

$$
\begin{aligned}
B_{x}= & \left\{-\frac{p_{1} k_{1} d_{1}\left(h_{1}-2\right)}{k_{1} d_{1}-a}, \frac{\left(-p_{1}+1\right) k_{1} d_{1}\left(h_{1}-2\right)}{k_{1} d_{1}-a}, \ldots,\right. \\
& \left.\frac{q_{1} k_{1} d_{1}\left(h_{1}-2\right)}{k_{1} d_{1}-a}\right\} .
\end{aligned}
$$

For all equilibria in set $A_{x}$, the characteristic equations are (7) and the corresponding eigenvalues satisfy $\lambda_{1}<0$ and $\lambda_{2,3}=$ $\alpha \pm \beta i$ with $\alpha>0$ and $\beta \neq 0$ from assumption (10). That is, all equilibria in set $A_{x}$ are saddle points of index 2. For all equilibria in set $B_{x}$, the corresponding characteristic equations are

$$
\lambda^{3}+c \lambda^{2}+b \lambda+a-d_{1} k_{1}=0 .
$$

Since $\lambda_{1}+\lambda_{2}+\lambda_{3}=-c<0$ and $\lambda_{1} \lambda_{2} \lambda_{3}=-\left(a-d_{1} k_{1}\right)>0$, (16) has one positive eigenvalue and two negative eigenvalues, or one positive eigenvalue and a pair of complex conjugate eigenvalues with negative real parts. To generate chaos from system (12), one may assume that (16) has a positive eigenvalue and a pair of complex eigenvalues with negative real parts. It means that all equilibria in set $B_{x}$ are saddle points of index 1 . Since the scrolls are generated only around saddle points of index 2 [17]-[19], system (12) has the potential to create a maximum of $\left(p_{1}+q_{1}+2\right)$-scroll chaotic attractor for some suitable parameters $a, b, c, d_{1}, k_{1}, h_{1}$. It should be emphasized that the $p_{1}+q_{1}+2$ equilibria in set $A_{x}$ are responsible for generating $p_{1}+q_{1}+2$ scrolls of the attractor. However, the $p_{1}+q_{1}+1$ equilibria in set $B_{x}$ are responsible for connecting these $p_{1}+q_{1}+2$ scrolls to form a whole chaotic attractor. Moreover, each equilibrium point in set $A_{x}$ corresponds to a unique saturated plateau of the saturated function series (5) and also corresponds to a unique scroll of the whole attractor. Furthermore, each equilibrium point in set $B_{x}$ corresponds to a unique saturated slope of the saturated function series (5) and also corresponds to a unique connection between two neighboring scrolls. 


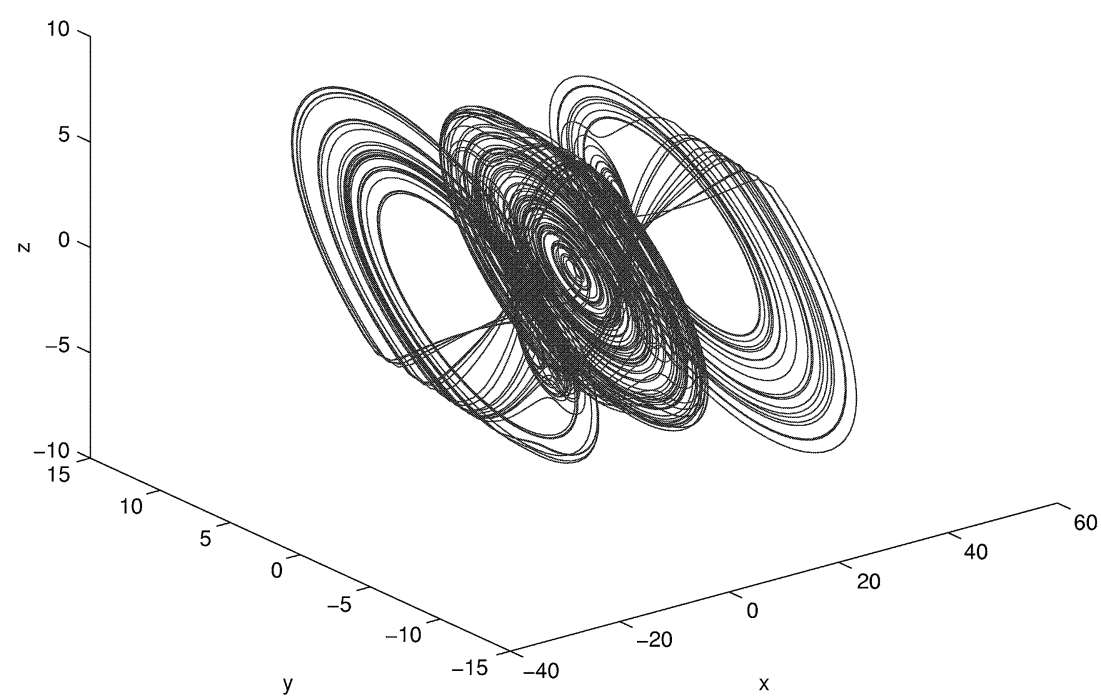

(a)

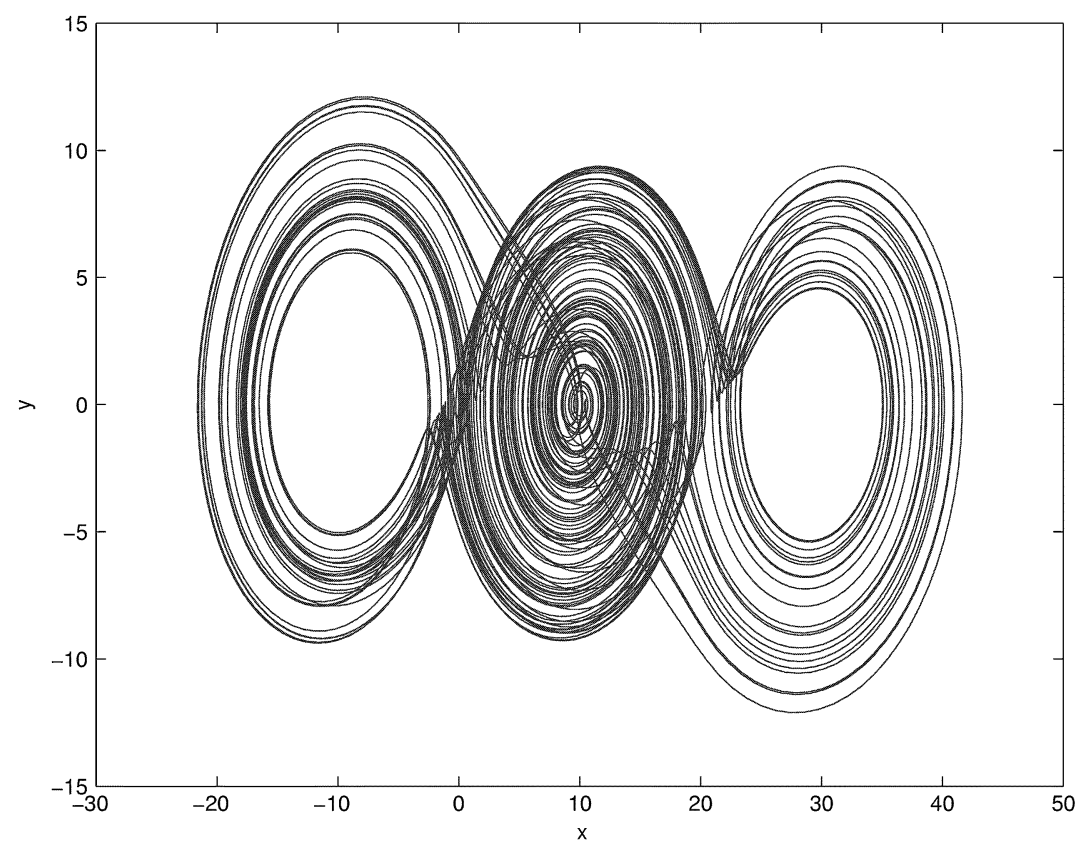

(b)

Fig. 5. Three-scroll chaotic attractor. (a) $x-y-z$ space. (b) $x-y$ plane projection.

Fig. 5 shows a 3 -scroll chaotic attractor of system (12), where $a=b=c=d_{1}=0.7, k_{1}=10, h_{1}=20, p_{1}=0, q_{1}=1$. The Lyapunov exponent spectrum of this 3-scroll chaotic attractor includes $\mathrm{LE}_{1}=0.1276, \mathrm{LE}_{2}=0, \mathrm{LE}_{3}=-0.8258$. Fig. 6 displays a 6-scroll chaotic attractor of system (12), where $a=$ $b=c=d_{1}=0.7, k_{1}=9, h_{1}=18, p_{1}=2, q_{1}=2$. The Lyapunov exponent spectrum of this 6-scroll chaotic attractor includes $\mathrm{LE}_{1}=0.1486, \mathrm{LE}_{2}=0, \mathrm{LE}_{3}=-0.8457$.

Remark 1: In fact, system (12) can create an $n$-scroll chaotic attractor $(n \geq 3)$, including odd and even number of scrolls chaotic attractors, by adjusting suitable parameters. There are three basic design strategies to obtain suitable circuit parameters for specified $n$-scroll chaotic attractors: 1) parameters $a, b, c$ satisfy condition (10); 2) parameters $a, d_{1}, k_{1}, h_{1}$ satisfy condition (13) where, for simplification, assume that $a=d_{1}$ and $\left.h_{1}=2 k_{1} ; 3\right)$ parameters $p_{1}, q_{1}$ control the number of scrolls in negative and positive $x$-directions, respectively.

\section{2-D $n \times m$-Grid Scroll Chaotic Attractors}

Here, a saturated function series controller is added to system (6) for generating $n \times m$-grid scroll chaotic attractors. The controlled system is described by

$$
\left\{\begin{aligned}
\dot{x}= & y-\frac{d_{2}}{b} f\left(y ; k_{2}, h_{2}, p_{2}, q_{2}\right) \\
\dot{y}= & z \\
\dot{z}= & -a x-b y-c z+d_{1} f\left(x ; k_{1}, h_{1}, p_{1}, q_{1}\right) \\
& \quad+d_{2} f\left(y ; k_{2}, h_{2}, p_{2}, q_{2}\right)
\end{aligned}\right.
$$

where $f\left(x ; k_{1}, h_{1}, p_{1}, q_{1}\right)$ and $f\left(y ; k_{2}, h_{2}, p_{2}, q_{2}\right)$ are defined by (5), and $a, b, c, d_{1}, d_{2}$ are positive constants.

Denote, in addition to (14) and (15), the following:

$$
\begin{aligned}
A_{y}= & \left\{-\frac{\left(2 p_{2}+1\right) d_{2} k_{2}}{b}, \frac{\left(-2 p_{2}+1\right) d_{2} k_{2}}{b}, \ldots,\right. \\
& \left.\frac{\left(2 q_{2}+1\right) d_{2} k_{2}}{b}\right\}
\end{aligned}
$$




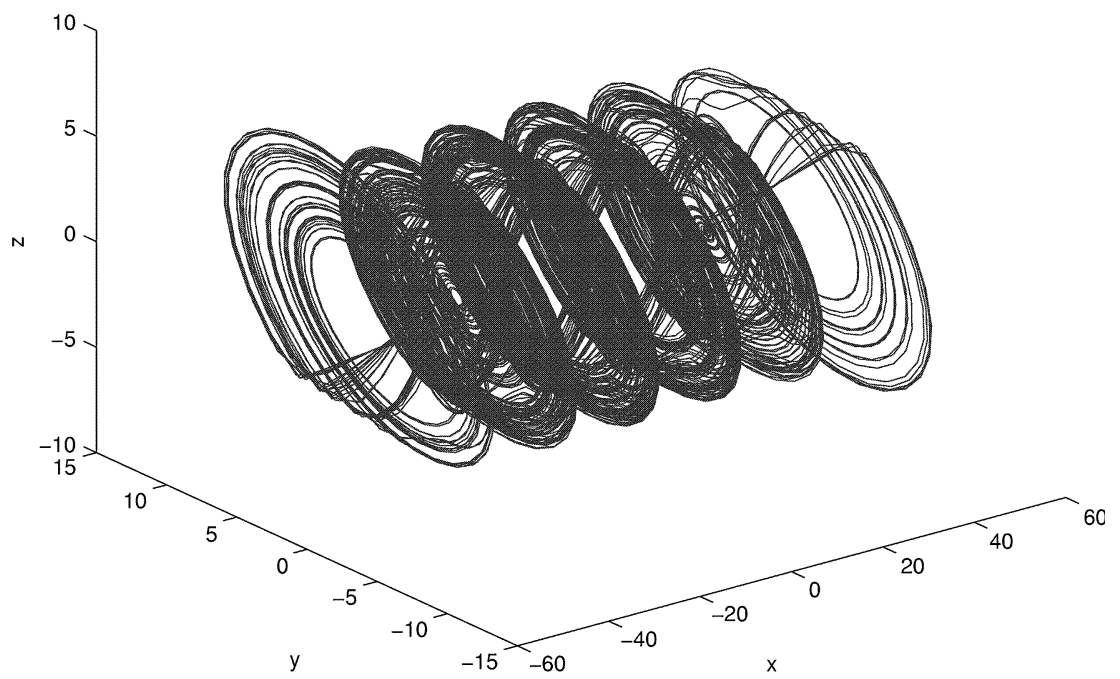

(a)

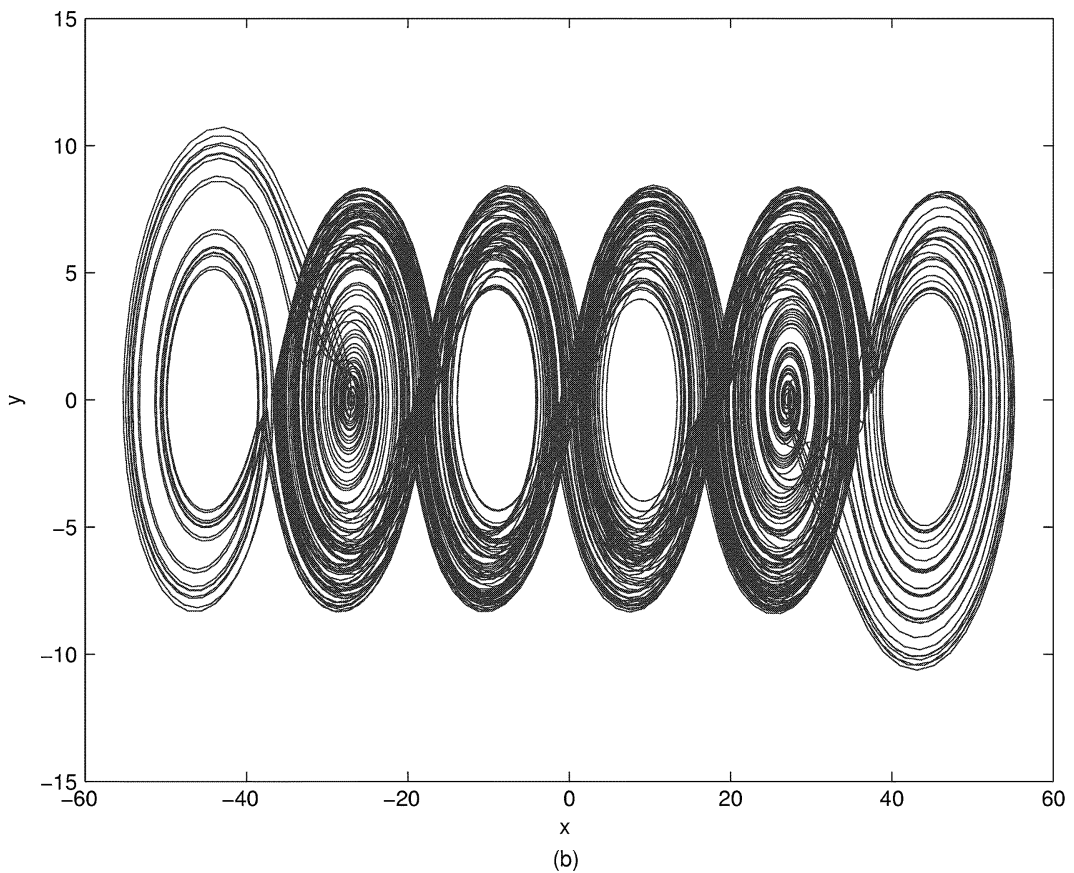

Fig. 6. Six-scroll chaotic attractor. (a) $x-y-z$ space. (b) $x-y$ plane projection.

and

$$
\begin{aligned}
B_{y}= & \left\{-\frac{p_{2} k_{2} d_{2}\left(h_{2}-2\right)}{k_{2} d_{2}-b}, \frac{\left(-p_{2}+1\right) k_{2} d_{2}\left(h_{2}-2\right)}{k_{2} d_{2}-b}, \ldots,\right. \\
& \left.\frac{q_{2} k_{2} d_{2}\left(h_{2}-2\right)}{k_{2} d_{2}-b}\right\} .
\end{aligned}
$$

Assume that (13) holds and

$$
\begin{array}{r}
d_{2} k_{2}>b, 2 d_{2} k_{2} \geq b h_{2}, \max \left\{p_{2}, q_{2}\right\} \frac{\left|b h_{2}-2 k_{2} d_{2}\right|}{d_{2} k_{2}-b} \leq 1, \\
\left(2 d_{2} k_{2}-b h_{2}\right)\left(q_{2}-1\right)<b h_{2}-d_{2} k_{2}-b .
\end{array}
$$

Then, (17) has $\left(2 p_{1}+2 q_{1}+3\right) \times\left(2 p_{2}+2 q_{2}+3\right)$ equilibrium points, which are located on the $x-y$ plane and given by

$$
O_{x y}=\left\{\left(x^{*}, y^{*}\right) \mid x^{*} \in A_{x} \cup B_{x}, \quad y^{*} \in A_{y} \cup B_{y}\right\} .
$$

It is noticed that all equilibria can be classified into four different sets

$$
\begin{aligned}
& A_{1}=\left\{\left(x^{*}, y^{*}\right) \mid x^{*} \in A_{x}, y^{*} \in A_{y}\right\} \\
& A_{2}=\left\{\left(x^{*}, y^{*}\right) \mid x^{*} \in A_{x}, y^{*} \in B_{y}\right\} \\
& A_{3}=\left\{\left(x^{*}, y^{*}\right) \mid x^{*} \in B_{x}, y^{*} \in A_{y}\right\} \\
& A_{4}=\left\{\left(x^{*}, y^{*}\right) \mid x^{*} \in B_{x}, y^{*} \in B_{y}\right\} .
\end{aligned}
$$

Obviously, the characteristic equations of the linearized system evaluated at the equilibria in set $A_{1}$ are (7) and the corresponding eigenvalues satisfy $\lambda_{1}<0$ and $\lambda_{2,3}=\alpha \pm \beta i$ with $\alpha>0$ and $\beta \neq 0$ by assumption (10). It means that all equilibria in set $A_{1}$ are saddle points of index 2 . For all equilibria in set $A_{2}$, the corresponding characteristic equations are

$$
\lambda^{3}+c \lambda^{2}+\left(b-k_{2} d_{2}\right) \lambda+a\left(1-\frac{k_{2} d_{2}}{b}\right)=0 .
$$


Since $\lambda_{1}+\lambda_{2}+\lambda_{3}=-c<0$ and $\lambda_{1} \lambda_{2} \lambda_{3}=-a(1-$ $\left.\left(k_{2} d_{2} / b\right)\right)>0,(22)$ has one positive eigenvalue and two negative eigenvalues, or one positive eigenvalue and a pair of complex conjugate eigenvalues with negative real parts. Moreover, all equilibria in $A_{2}$ are saddle points of index 1 . For all equilibria in $A_{3}$, the corresponding characteristic equations are (16) and these equilibria are saddle points of index 1 . Similarly, for all equilibria in $A_{4}$, the corresponding characteristic equations are

$$
\lambda^{3}+c \lambda^{2}+\left(b-k_{2} d_{2}\right) \lambda+\left(a-d k_{1}\right)\left(1-\frac{k_{2} d_{2}}{b}\right)=0
$$

Since $\lambda_{1}+\lambda_{2}+\lambda_{3}=-c<0$ and $\lambda_{1} \lambda_{2} \lambda_{3}=-\left(a-d k_{1}\right)(1-$ $\left.\left(k_{2} d_{2}\right) /(b)\right)<0$, (23) has one negative eigenvalue and two positive eigenvalues, or three negative eigenvalues, or one negative eigenvalue and a pair of complex conjugate eigenvalues.

To create chaos from system (17), one may assume that (23) has one negative eigenvalue and a pair of complex conjugate eigenvalues with positive real parts. Thus, the equilibria in $A_{4}$ are saddle points of index 2 . Since the scrolls can be generated only around saddle points of index 2 [17]-[19], the equilibria in $A_{1}$ and $A_{4}$ may create scrolls. However, numerical simulations show that only the equilibria in $A_{1}$ can generate scrolls. In fact, having a saddle point of index 2 is only a necessary condition, but not a sufficient condition for generating scrolls. According to the Homoclinic Šilnikov Theorem [19], it needs a condition-existence of a homoclinic orbit in the neighboring region of the equilibrium point-for generating scrolls. Therefore, system (17) has the potential to create a maximum of 2-D $\left(p_{1}+q_{1}+2\right) \times\left(p_{2}+q_{2}+2\right)$-grid scroll chaotic attractor, called 2-D $n \times m$-grid scroll chaotic attractor, for suitable parameters $a, b, c, d_{1}, d_{2}, k_{1}, h_{1}, k_{2}, h_{2}$. Note that each equilibrium point in $A_{1}$ corresponds to a unique 2-D saturated plateau and also corresponds to a unique scroll in the whole attractor. Moreover, other equilibria in $A_{2}, A_{3}, A_{4}$ correspond to the saturated slopes and are responsible for connecting these $\left(p_{1}+q_{1}+2\right) \times$ $\left(p_{2}+q_{2}+2\right)$ scrolls.

Fig. 7 shows a $6 \times 6$-grid scroll chaotic attractor, where $a=$ $b=c=d_{1}=d_{2}=0.7, k_{1}=k_{2}=50, h_{1}=h_{2}=100, p_{1}=$ $q_{1}=p_{2}=q_{2}=2$. Clearly, there are six scrolls in the $x$-direction and six scrolls in the $y$-direction, as shown in Fig. 7(b). The Lyapunov exponent spectrum of this $6 \times 6$-grid scroll attractor includes $\mathrm{LE}_{1}=0.1599, \mathrm{LE}_{2}=0, \mathrm{LE}_{3}=-0.8622$. Note that these 2-D $n \times m$-grid scroll chaotic attractors are generated in exactly the same way as the 1-D case discussed in the last subsection, except that the directions of the system trajectories are more vertical here. Similarly, one can design 2-D $n \times m$-grid scroll attractors in $x-z$ or $y-z$ directions.

Remark 2: There are five fundamental design guidelines for obtaining suitable circuit parameters for specified 2-D $n \times m$-grid scroll attractors: 1) parameters $a, b, c$ satisfy condition (10); 2) parameters $a, d_{1}, k_{1}, h_{1}$ satisfy condition (13) where, for simplification, assume that $a=d_{1}$ and $h_{1}=2 k_{1} ; 3$ ) parameters $b, d_{2}, k_{2}, h_{2}$ satisfy condition (20) where, for simplification, assume that $b=d_{2}$ and $h_{2}=2 k_{2} ; 4$ ) parameters $p_{1}, q_{1}$ control the numbers of scrolls in negative and positive $x$-directions, respectively; 5) parameters $p_{2}, q_{2}$ control the numbers of scrolls in negative and positive $y$-directions, respectively.

\section{3-D $n \times m \times l$-Grid Scroll Chaotic Attractors}

Here, a saturated function series controller is added to system (6) for creating 3-D $n \times m \times l$-grid scroll chaotic attractors. The controlled system is

$$
\left\{\begin{aligned}
\dot{x}= & y-\frac{d_{2}}{b} f\left(y ; k_{2}, h_{2}, p_{2}, q_{2}\right) \\
\dot{y}= & z-\frac{d_{3}}{c} f\left(z ; k_{3}, h_{3}, p_{3}, q_{3}\right) \\
\dot{z}= & -a x-b y-c z+d_{1} f\left(x ; k_{1}, h_{1}, p_{1}, q_{1}\right) \\
& \quad+d_{2} f\left(y ; k_{2}, h_{2}, p_{2}, q_{2}\right)+d_{3} f\left(z ; k_{3}, h_{3}, p_{3}, q_{3}\right)
\end{aligned}\right.
$$

with $f\left(x ; k_{1}, h_{1}, p_{1}, q_{1}\right), f\left(y ; k_{2}, h_{2}, p_{2}, q_{2}\right) f\left(z ; k_{3}, h_{3}, p_{3}, q_{3}\right)$ defined by (5), and $a, b, c, d_{1}, d_{2}, d_{3}$ are positive constants.

In addition to (14), (15), (18), and (19), define

$$
\begin{aligned}
A_{z}= & \left\{-\frac{\left(2 p_{3}+1\right) d_{3} k_{3}}{c}, \frac{\left(-2 p_{3}+1\right) d_{3} k_{3}}{c}, \ldots,\right. \\
& \left.\frac{\left(2 q_{3}+1\right) d_{3} k_{3}}{c}\right\}
\end{aligned}
$$

and

$$
\begin{aligned}
B_{z}= & \left\{-\frac{p_{3} k_{3} d_{3}\left(h_{3}-2\right)}{k_{3} d_{3}-c}, \frac{\left(-p_{3}+1\right) k_{3} d_{3}\left(h_{3}-2\right)}{k_{3} d_{3}-c}, \ldots,\right. \\
& \left.\frac{q_{3} k_{3} d_{3}\left(h_{3}-2\right)}{k_{3} d_{3}-c}\right\} .
\end{aligned}
$$

Assume that (13) and (20) hold and

$$
\begin{aligned}
& d_{3} k_{3}>c, \quad 2 d_{3} k_{3} \geq c h_{3}, \max \left\{p_{3}, q_{3}\right\} \frac{\left|c h_{3}-2 k_{3} d_{3}\right|}{d_{3} k_{3}-c} \leq 1 \\
&\left(2 d_{3} k_{3}-c h_{3}\right)\left(q_{3}-1\right)<c h_{3}-d_{3} k_{3}-c .
\end{aligned}
$$

Then, (24) has $\left(2 p_{1}+2 q_{1}+3\right) \times\left(2 p_{2}+2 q_{2}+3\right) \times\left(2 p_{3}+2 q_{3}+3\right)$ equilibrium points, given by

$$
\begin{aligned}
O_{x y z}=\left\{\left(x^{*}, y^{*}, z^{*}\right) \mid x^{*}\right. & \in A_{x} \cup B_{x}, \\
y^{*} & \left.\in A_{y} \cup B_{y}, z^{*} \in A_{z} \cup B_{z}\right\} .
\end{aligned}
$$

Note that all equilibria can be classified into eight different sets:

$$
\begin{aligned}
& \bar{A}_{1}=\left\{\left(x^{*}, y^{*}, z^{*}\right) \mid x^{*} \in A_{x}, y^{*} \in A_{y}, z^{*} \in A_{z}\right\} \\
& \bar{A}_{2}=\left\{\left(x^{*}, y^{*}, z^{*}\right) \mid x^{*} \in A_{x}, y^{*} \in A_{y}, z^{*} \in B_{z}\right\} \\
& \bar{A}_{3}=\left\{\left(x^{*}, y^{*}, z^{*}\right) \mid x^{*} \in A_{x}, y^{*} \in B_{y}, z^{*} \in A_{z}\right\} \\
& \bar{A}_{4}=\left\{\left(x^{*}, y^{*}, z^{*}\right) \mid x^{*} \in A_{x}, y^{*} \in B_{y}, z^{*} \in B_{z}\right\} \\
& \bar{A}_{5}=\left\{\left(x^{*}, y^{*}, z^{*}\right) \mid x^{*} \in B_{x}, y^{*} \in A_{y}, z^{*} \in A_{z}\right\} \\
& \bar{A}_{6}=\left\{\left(x^{*}, y^{*}, z^{*}\right) \mid x^{*} \in B_{x}, y^{*} \in A_{y}, z^{*} \in B_{z}\right\} \\
& \bar{A}_{7}=\left\{\left(x^{*}, y^{*}, z^{*}\right) \mid x^{*} \in B_{x}, y^{*} \in B_{y}, z^{*} \in A_{z}\right\} \\
& \bar{A}_{8}=\left\{\left(x^{*}, y^{*}, z^{*}\right) \mid x^{*} \in B_{x}, y^{*} \in B_{y}, z^{*} \in B_{z}\right\} .
\end{aligned}
$$

For all equilibria in $\bar{A}_{1}$, the corresponding characteristic equations are (7). From assumption (10), all equilibria in $\bar{A}_{1}$ are saddle points of index 2 . For the equilibrium points in $\bar{A}_{2}$, the corresponding characteristic equations are

$$
\lambda^{3}+\left(c-d_{3} k_{3}\right) \lambda^{2}+b\left(1-\frac{k_{3} d_{3}}{c}\right) \lambda+a\left(1-\frac{k_{3} d_{3}}{c}\right)=0 .
$$

Since $\lambda_{1}+\lambda_{2}+\lambda_{3}=-\left(c-d_{3} k_{3}\right)>0$ and $\lambda_{1} \lambda_{2} \lambda_{3}=-a(1-$ $\left.\left(k_{3} d_{3}\right) /(c)\right)>0$, (29) has three positive eigenvalues, or one positive eigenvalue and two negative eigenvalues, or one positive eigenvalue and a pair of complex conjugate eigenvalues. Based on numerical observations, one may assume that (29) 

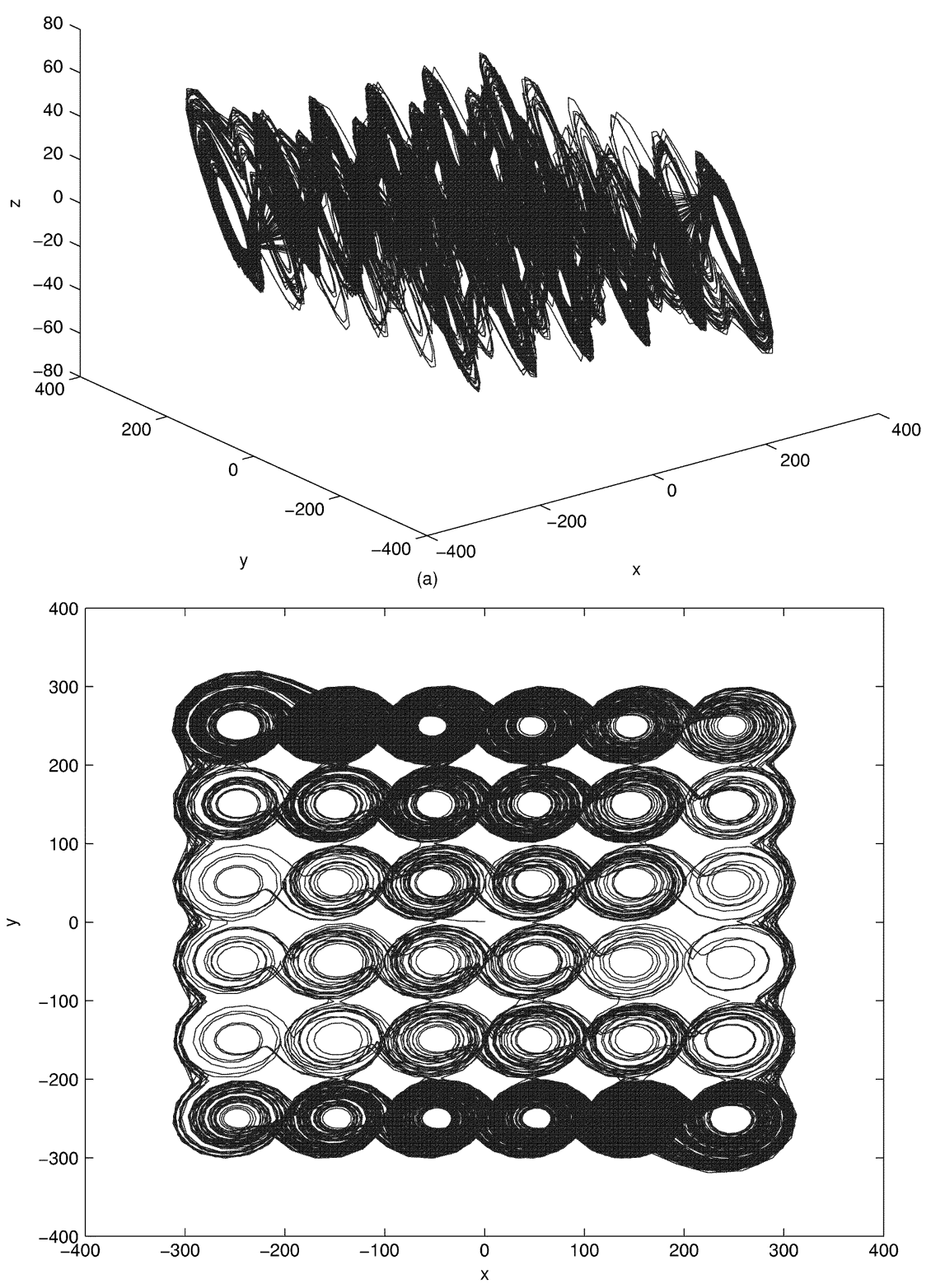

(b)

Fig. 7. Two-directional $6 \times 6$-grid scroll chaotic attractors. (a) $x-y-z$ space.(b) $x-y$ plane projection.

has one positive eigenvalue and a pair of complex conjugate eigenvalues with negative real parts. Thus, all equilibria in $\bar{A}_{2}$ are saddle points of index 1 . For all equilibria in $\bar{A}_{3}$, the corresponding characteristic equations are (22).

According to the assumption in the last subsection, the equilibria in $\bar{A}_{3}$ are saddle points of index 1 . For the equilibria in $\bar{A}_{4}$, the corresponding characteristic equations are

$$
\begin{aligned}
\lambda^{3}+\left(c-d_{3} k_{3}\right) \lambda^{2} & +\left(b-d_{2} k_{2}\right)\left(1-\frac{k_{3} d_{3}}{c}\right) \lambda \\
& +a\left(1-\frac{k_{2} d_{2}}{b}\right)\left(1-\frac{k_{3} d_{3}}{c}\right)=0 .
\end{aligned}
$$

Since $\lambda_{1}+\lambda_{2}+\lambda_{3}=-\left(c-d_{3} k_{3}\right)>0$ and $\lambda_{1} \lambda_{2} \lambda_{3}=$ $-a\left(1-\left(\left(k_{2} d_{2}\right) /(b)\right)\left(1-\left(k_{3} d_{3}\right) /(c)\right)<0,(30)\right.$ has one negative eigenvalue and two positive eigenvalues, or one negative eigenvalue and a pair of complex conjugate eigenvalues with positive real parts. Numerical observations show that (30) has one negative eigenvalue and a pair of complex conjugate eigenvalues with positive real parts. Thus, the equilibria in $\bar{A}_{4}$ are saddle points of index 2 . For all equilibria in $\bar{A}_{5}$, the corresponding characteristic equations are (16). According to the assumption in Subsection B, all equilibria in $\bar{A}_{5}$ are saddle points of index 1 . For the equilibria in $\bar{A}_{6}$, the corresponding characteristic equations are

$$
\begin{aligned}
\lambda^{3}+\left(c-d_{3} k_{3}\right) \lambda^{2}+b & \left(1-\frac{k_{3} d_{3}}{c}\right) \lambda \\
& +\left(a-k_{1} d_{1}\right)\left(1-\frac{k_{3} d_{3}}{c}\right)=0 .
\end{aligned}
$$

Since $\lambda_{1}+\lambda_{2}+\lambda_{3}=-\left(c-d_{3} k_{3}\right)>0$ and $\lambda_{1} \lambda_{2} \lambda_{3}=$ $-\left(a-k_{1} d_{1}\right)\left(1-\left(k_{3} d_{3}\right) /(c)\right)<0,(31)$ has one negative eigenvalue and two positive eigenvalues, or one negative eigenvalue and a pair of complex conjugate eigenvalues with positive real 


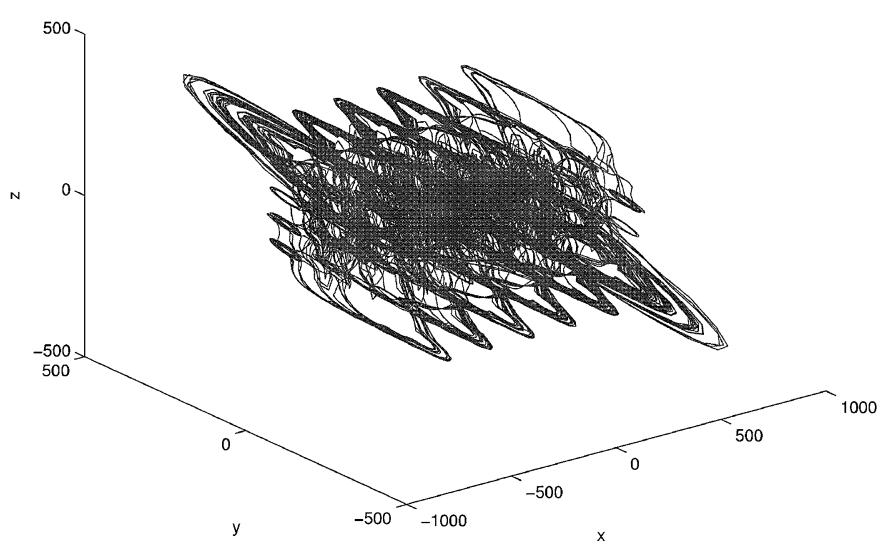

(a)

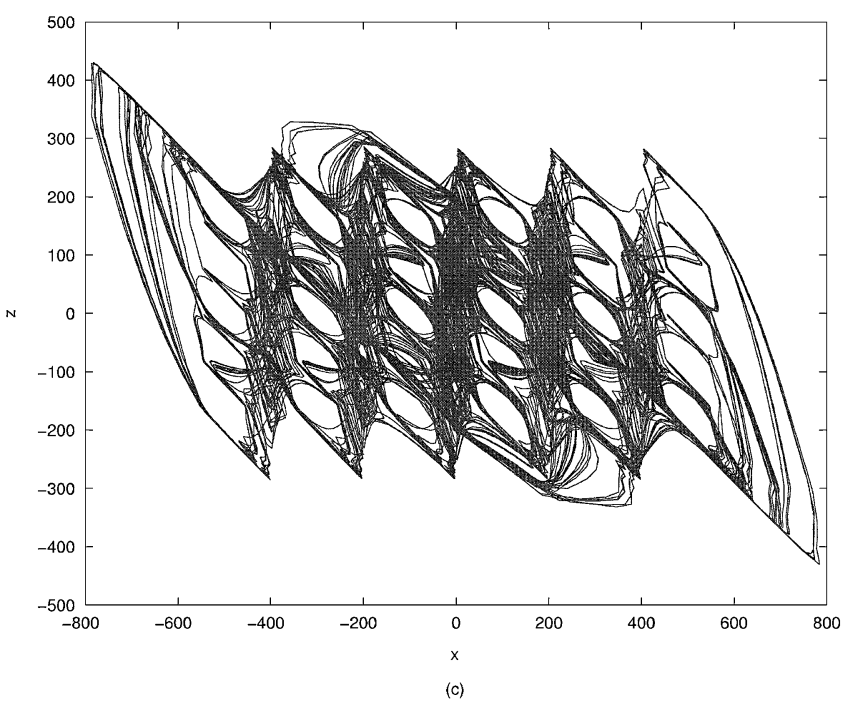

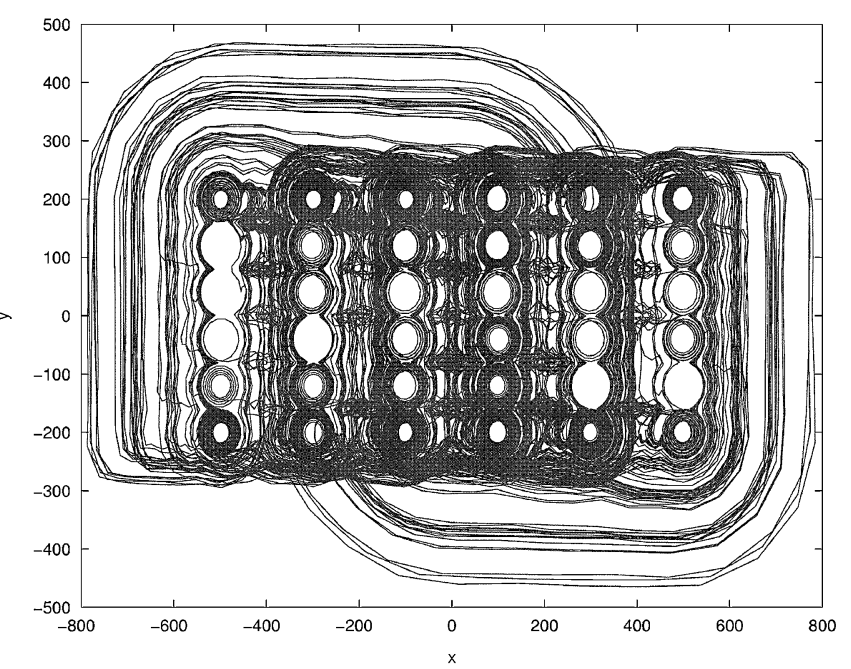

(b)

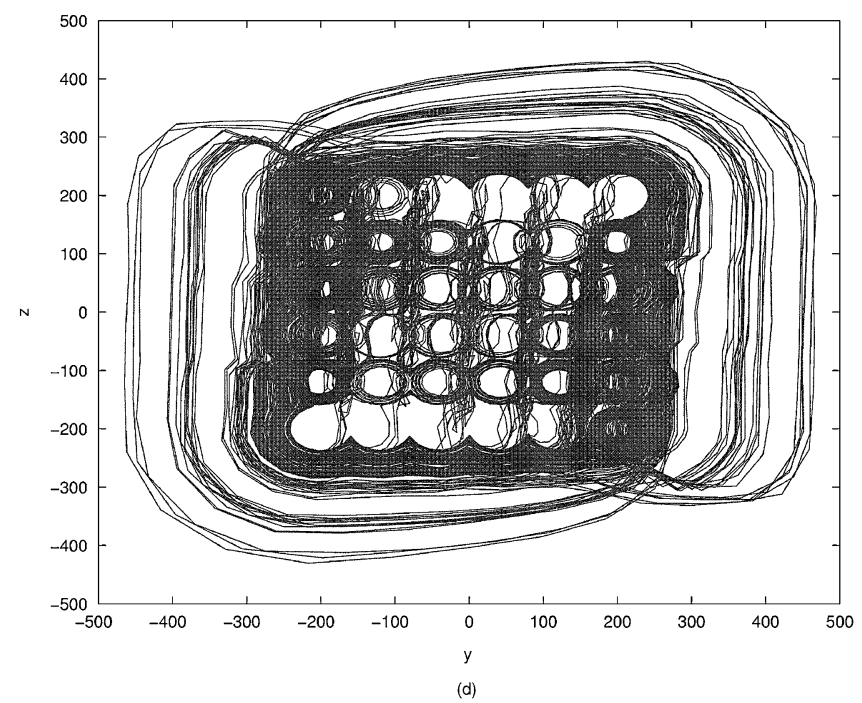

Fig. 8. Three-directional $6 \times 6 \times 6$-grid scroll chaotic attractors. (a) $x-y-z$ space. (b) $x-y$ plane projection. (c) $x-z$ plane projection. (d) $y-z$ plane projection.

parts. Numerical simulations show that (31) has one negative eigenvalue and two positive eigenvalues. So the equilibria in $\bar{A}_{6}$ are saddle points of index 1 . For all equilibria in $\bar{A}_{7}$, the corresponding characteristic equations are (23). From the assumption in the last subsection, all equilibria in $\bar{A}_{7}$ are saddle points of index 2 . Finally, consider all equilibria in $\bar{A}_{8}$, the corresponding characteristic equations are

$$
\begin{aligned}
\lambda^{3}+(c- & \left.d_{3} k_{3}\right) \lambda^{2}+\left(b-d_{2} k_{2}\right)\left(1-\frac{k_{3} d_{3}}{c}\right) \lambda \\
& +\left(a-k_{1} d_{1}\right)\left(1-\frac{k_{2} d_{2}}{b}\right)\left(1-\frac{k_{3} d_{3}}{c}\right)=0 .
\end{aligned}
$$

Since $\lambda_{1}+\lambda_{2}+\lambda_{3}=-\left(c-d_{3} k_{3}\right)>0$ and $\lambda_{1} \lambda_{2} \lambda_{3}=$ $-\left(a-d_{1} k_{1}\right)\left(1-\left(k_{2} d_{2}\right) /(b)\right)\left(1-\left(k_{3} d_{3}\right) /(c)\right)>0$, (32) has three positive eigenvalues, or one positive eigenvalue and two negative eigenvalues, or one positive eigenvalue and a pair of complex conjugate eigenvalues. Numerical observations show that (32) has one positive eigenvalue and a pair of complex conjugate eigenvalues with negative real parts. Then, the equilibria in $\bar{A}_{8}$ are saddle points of index 1 .
It should be pointed out that the scrolls can be generated only around saddle points of index 2 [17]-[19]. Therefore, only the equilibria in $\bar{A}_{1}, \bar{A}_{4}$, and $\bar{A}_{7}$ may create scrolls. However, numerical observations reveal that only the equilibria in $\bar{A}_{1}$ can generate scrolls. In fact, having a saddle point of index 2 is only a necessary condition, but not a sufficient condition for generating scrolls. That is, system (24) has the potential to create a maximum of 3-D $\left(p_{1}+q_{1}+2\right) \times\left(p_{2}+q_{2}+2\right) \times\left(p_{3}+\right.$ $q_{3}+2$ )-grid scroll chaotic attractor, called 3-D $n \times m \times l$ grid scroll chaotic attractor, for some suitable parameters $a, b, c$, $d_{1}, d_{2}, d_{3}, k_{1}, h_{1}, k_{2}, h_{2}, k_{3}, h_{3}$. In particular, each equilibrium point in $\bar{A}_{1}$ corresponds to a unique 3-D saturated plateau and also corresponds to a unique scroll in the whole attractor. Furthermore, other equilibria in $\bar{A}_{i}(2 \leq i \leq 8)$ correspond to the saturated slopes and are responsible for connecting the $\left(p_{1}+\right.$ $\left.q_{1}+2\right) \times\left(p_{2}+q_{2}+2\right) \times\left(p_{3}+q_{3}+2\right)$ scrolls.

Fig. 8 shows a $6 \times 6 \times 6$-grid scroll chaotic attractor, where $a=d_{1}=0.7, b=c=d_{2}=d_{3}=0.8, k_{1}=100, h_{1}=$ $200, k_{2}=k_{3}=40, h_{2}=h_{3}=80, p_{1}=p_{2}=p_{3}=q_{1}=q_{2}=$ $q_{3}=2$. Obviously, there are six scrolls in each direction of the state space, as shown in Fig. 8(b), (c), and (d), respectively. 
The Lyapunov exponent spectrum of this $6 \times 6 \times 6$-grid scroll attractor includes $\mathrm{LE}_{1}=0.0885, \mathrm{LE}_{2}=0, \mathrm{LE}_{3}=-0.7157$. Note that these 3 -D $n \times m \times l$-grid scroll chaotic attractors are generated in exactly the same way as the 1-D and 2-D cases discussed before, except that the directions of the system trajectories are three here.

Remark 3: There are seven basic design strategies to obtain suitable circuit parameters for specified 3-D $n \times m \times l$-grid scroll attractors: 1) parameters $a, b, c$ satisfy condition (10); 2) parameters $a, d_{1}, k_{1}, h_{1}$ satisfy condition (13) where, for simplification, assume that $a=d_{1}$ and $h_{1}=2 k_{1} ; 3$ ) parameters $b, d_{2}, k_{2}, h_{2}$ satisfy condition (20) where, for simplification, assume that $b=$ $d_{2}$ and $\left.h_{2}=2 k_{2} ; 4\right)$ parameters $c, d_{3}, k_{3}, h_{3}$ satisfy condition (27) where, for simplification, assume that $c=d_{3}$ and $h_{3}=2 k_{3}$; 5) parameters $p_{1}, q_{1}$ control the numbers of scrolls in negative and positive $x$-directions, respectively; 6 ) parameters $p_{2}, q_{2}$ control the number of scrolls in negative and positive $y$-directions, respectively; 7) parameters $p_{3}, q_{3}$ control the number of scrolls in negative and positive $z$-directions, respectively.

\section{THEORETICAL ANALYSIS FOR MULTISCROLL CHAOTIC ATTRACTORS}

\section{A. Dynamical Analysis of Double-Scroll System (11)}

Here, the dynamical behaviors of the double-scroll systems (11) are further investigated. In particular, a 2-D Poincaré return map [18], [26] is rigorously derived for verifying the chaotic behaviors of the double-scroll system (11).

Obviously, systems (12), (17), and (24) become system (11) in every corresponding subspace. Therefore, system (11) is the basic generator of the multiscroll chaotic systems (12), (17), and (24) and plays a very important role in chaos generation. When $a=b=c=d_{1}=0.7, k=10$, system (11) has a double-scroll chaotic attractor as shown in Fig. 4. Obviously, (11) has three different subspaces

$$
\begin{aligned}
& V_{1}=\{\mathbf{X} \mid x \geq 1\} \\
& V_{2}=\{\mathbf{X}|| x \mid \leq 1\} \\
& V_{3}=\{\mathbf{X} \mid x \leq-1\}
\end{aligned}
$$

where $\mathbf{X}=(x, y, z)^{T}$. System (11) has a natural symmetry under the coordinates transform $(x, y, z) \rightarrow(-x,-y,-z)$, which persists for all parameter values. The variation of the volume $V(t)$ of a small element, $\delta V(t)=\delta x \delta y \delta z$, in the state space is determined by the divergence of the flow

$$
\nabla V=\frac{\partial \dot{x}}{\partial x}+\frac{\partial \dot{y}}{\partial y}+\frac{\partial \dot{z}}{\partial z}=-c<0
$$

That is, system (11) is dissipative in each subspace.
For subspaces $V_{1}$ and $V_{3}$, the dynamical equations are described by

$$
\left(\begin{array}{c}
\dot{\bar{x}} \\
\overline{\bar{y}} \\
\dot{\bar{z}}
\end{array}\right)=\left(\begin{array}{ccc}
0 & 1 & 0 \\
0 & 0 & 1 \\
-a & -b & -c
\end{array}\right)\left(\begin{array}{c}
\bar{x} \\
\bar{y} \\
\bar{z}
\end{array}\right)
$$

where

$$
\begin{array}{ll}
(\bar{x}, \bar{y}, \bar{z})^{T}=\left(x-\frac{d_{1} k_{1}}{a}, y, z\right)^{T} \quad \text { for } \mathbf{X} \in V_{1}, \\
(\bar{x}, \bar{y}, \bar{z})^{T}=\left(x+\frac{d_{1} k_{1}}{a}, y, z\right)^{T} \quad \text { for } \mathbf{X} \in V_{3} .
\end{array}
$$

Thus, the exact solution of the above equation is

$$
\left\{\begin{aligned}
\bar{x}(t)= & A_{1} e^{\lambda_{1} t}+e^{\alpha t}\left(A_{2} \cos (\beta t)+A_{3} \sin (\beta t)\right) \\
\bar{y}(t)= & A_{1} \lambda_{1} e^{\lambda_{1} t}+e^{\alpha t}\left[\left(A_{2} \alpha+A_{3} \beta\right) \cos (\beta t)\right. \\
& \left.+\left(A_{3} \alpha-A_{2} \beta\right) \sin (\beta t)\right] \\
\bar{z}(t)= & A_{1} \lambda_{1}^{2} e^{\lambda_{1} t} \\
& +e^{\alpha t}\left[\left(A_{2} \alpha^{2}+2 A_{3} \alpha \beta-A_{2} \beta^{2}\right) \cos (\beta t)\right. \\
& \left.+\left(A_{3} \alpha^{2}-2 A_{2} \alpha \beta-A_{3} \beta^{2}\right) \sin (\beta t)\right]
\end{aligned}\right.
$$

where coefficients are given at the bottom of the page, and $\lambda_{1}, \alpha, \beta$ are defined by (8) and (9). Denote solution (33) as

$$
\left(\begin{array}{c}
\bar{x}(t) \\
\bar{y}(t) \\
\bar{z}(t)
\end{array}\right)=\left(\begin{array}{l}
g_{1}(t) \\
g_{2}(t) \\
g_{3}(t)
\end{array}\right)\left(\begin{array}{l}
\bar{x}_{0} \\
\bar{y}_{0} \\
\bar{z}_{0}
\end{array}\right)
$$

For subspace $V_{3}$, the dynamical equation is described by

$$
\left(\begin{array}{c}
\dot{x} \\
\dot{y} \\
\dot{z}
\end{array}\right)=\left(\begin{array}{ccc}
0 & 1 & 0 \\
0 & 0 & 1 \\
d_{1} k_{1}-a & -b & -c
\end{array}\right)\left(\begin{array}{l}
x \\
y \\
z
\end{array}\right) .
$$

Clearly, the exact solution is

$$
\left\{\begin{aligned}
x(t)= & A_{1}^{\prime} e^{\lambda_{1}^{\prime} t}+e^{\alpha^{\prime} t}\left(A_{2}^{\prime} \cos \left(\beta^{\prime} t\right)+A_{3}^{\prime} \sin \left(\beta^{\prime} t\right)\right) \\
y(t)= & A_{1}^{\prime} \lambda_{1}^{\prime} e^{\lambda_{1}^{\prime} t}+e^{\alpha^{\prime} t}\left[\left(A_{2}^{\prime} \alpha^{\prime}+A_{3}^{\prime} \beta^{\prime}\right) \cos \left(\beta^{\prime} t\right)\right. \\
& \left.+\left(A_{3}^{\prime} \alpha^{\prime}-A_{2}^{\prime} \beta^{\prime}\right) \sin \left(\beta^{\prime} t\right)\right] \\
z(t)= & A_{1}^{\prime} \lambda_{1}^{\prime 2} e^{\lambda_{1}^{\prime} t}+e^{\alpha^{\prime} t}\left[\left(A_{2}^{\prime} \alpha^{\prime 2}\right.\right. \\
& \left.+2 A_{3}^{\prime} \alpha^{\prime} \beta^{\prime}-A_{2}^{\prime} \beta^{\prime 2}\right) \cos \left(\beta^{\prime} t\right) \\
& \left.+\left(A_{3}^{\prime} \alpha^{\prime 2}-2 A_{2}^{\prime} \alpha^{\prime} \beta^{\prime}-A_{3}^{\prime} \beta^{\prime 2}\right) \sin \left(\beta^{\prime} t\right)\right]
\end{aligned}\right.
$$

where coefficients are given at the bottom of the next page, in which $\lambda_{1}^{\prime}$ is the positive eigenvalue of (16), and $\alpha^{\prime}, \beta^{\prime}$ are the real and imaginary parts of the pair of complex conjugate eigenvalues of (16). Denote solution (34) as

$$
\left(\begin{array}{l}
x(t) \\
y(t) \\
z(t)
\end{array}\right)=\left(\begin{array}{l}
g_{1}^{\prime}(t) \\
g_{2}^{\prime}(t) \\
g_{3}^{\prime}(t)
\end{array}\right)\left(\begin{array}{l}
x_{0} \\
y_{0} \\
z_{0}
\end{array}\right)
$$

$$
\begin{aligned}
& A_{1}=\frac{\left(\alpha^{2}+\beta^{2}\right) \bar{x}(0)-2 \alpha \bar{y}(0)+\bar{z}(0)}{\left(\lambda_{1}-\alpha\right)^{2}+\beta^{2}} \\
& A_{2}=\frac{\left(\lambda_{1}^{2}-2 \alpha \lambda_{1}\right) \bar{x}(0)+2 \alpha \bar{y}(0)-\bar{z}(0)}{\left(\lambda_{1}-\alpha\right)^{2}+\beta^{2}} \\
& A_{3}=\frac{\left(\lambda_{1} \alpha^{2}-\lambda_{1} \beta^{2}-\lambda_{1}^{2} \alpha\right) \bar{x}(0)-\left(\beta^{2}-\alpha^{2}+\lambda_{1}^{2}\right) \bar{y}(0)+\left(\alpha-\lambda_{1}\right) \bar{z}(0)}{\beta\left[\left(\lambda_{1}-\alpha\right)^{2}+\beta^{2}\right]}
\end{aligned}
$$




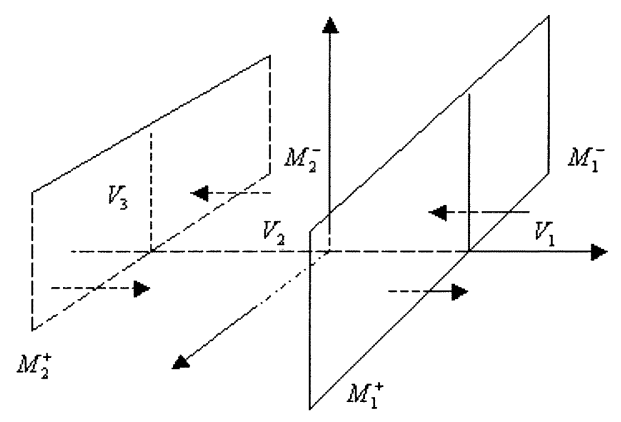

Fig. 9. Trajectory switchings of the double-scroll system (11).

Obviously, system (11) has two symmetrical switching planes: $M_{1} \equiv\{\mathbf{X} \mid x=1\}$ and $M_{2} \equiv\{\mathbf{X} \mid x=-1\}$. Denote

$$
\begin{aligned}
& M_{1}^{+} \equiv\{\mathbf{X} \mid x=1, y>0\} \quad M_{1}^{-} \equiv\{\mathbf{X} \mid x=1, y<0\} \\
& M_{2}^{+} \equiv\{\mathbf{X} \mid x=-1, y>0\} \quad M_{2}^{-} \equiv\{\mathbf{X} \mid x=-1, y<0\} .
\end{aligned}
$$

and the corresponding switching rules are

$$
\begin{aligned}
& \mathbf{X}\left(t^{+}\right)=(1, y, z)^{T} \in V_{2} \quad \text { if } \mathbf{X}(t)=(1, y, z)^{T} \in M_{1}^{-} \\
& \mathbf{X}\left(t^{+}\right)=(-1, y, z)^{T} \in V_{3} \quad \text { if } \mathbf{X}(t)=(-1, y, z)^{T} \in M_{2}^{-} \\
& \mathbf{X}\left(t^{+}\right)=(-1, y, z)^{T} \in V_{2} \quad \text { if } \mathbf{X}(t)=(-1, y, z)^{T} \in M_{2}^{+} \\
& \mathbf{X}\left(t^{+}\right)=(1, y, z)^{T} \in V_{1} \quad \text { if } \mathbf{X}(t)=(1, y, z)^{T} \in M_{1}^{+}
\end{aligned}
$$

where $\mathbf{X}=(x, y, z)^{T}$.

Fig. 9 shows the trajectory switchings of system (11). Consider switching plane $M_{1}$. If $y>0, \dot{x}=y>0$, the trajectories of system (11) will go through switching plane $M_{1}^{+}$from $V_{2}$ to $V_{1}$; if $y<0, \dot{x}=y<0$, the trajectories of system (11) will go through switching plane $M_{1}^{-}$from $V_{1}$ to $V_{2}$. For switching plane $M_{2}$, if $y>0, \dot{x}=y>0$, the trajectories of system (11) will go through switching plane $M_{2}^{+}$from $V_{3}$ to $V_{2}$; if $y<$ $0, \dot{x}=y<0$, the trajectories of system (11) will go through switching plane $M_{2}^{-}$from $V_{2}$ to $V_{3}$. Without loss of generality, assume that the initial point $x_{0} \in V_{1}$. Then, after a long enough time, it will definitely arrive at the switching plane $M_{1}^{-}$. From the switching rules, this trajectory will go through $M_{1}^{-}$from $V_{1}$ to $V_{2}$. After a long enough time, it will definitely reach $M_{1}^{+}$ or $M_{2}^{-}$; if it arrives at $M_{1}^{+}$, it will go through switching plane $M_{1}^{+}$from $V_{2}$ to $V_{1}$ and begin a new cycle; if it reaches $M_{2}^{-}$, according to the switching rules, it will go through switching plane $M_{2}^{-}$from $V_{2}$ to $V_{3}$. After a long enough time, this trajectory will definitely arrive at switching plane $M_{2}^{+}$; from the switching rules, this trajectory will go through switching plane $M_{2}^{+}$from
$V_{3}$ to $V_{2}$. After some time, it will reach switching plane $M_{2}^{-}$or $M_{1}^{+}$; if it arrives at $M_{2}^{-}$, it will repeat the former process; if it reaches $M_{1}^{+}$, according to the switching rules, it will go through the switching plane $M_{1}^{+}$from $V_{2}$ to $V_{1}$ and begin a new cycle. Therefore, the running routes of this trajectory can be classified into three different cases

$$
\begin{aligned}
V_{1} & \rightarrow M_{1}^{-} \rightarrow V_{2} \rightarrow M_{1}^{+} \rightarrow V_{1} \\
V_{1} & \rightarrow M_{1}^{-} \rightarrow V_{2} \rightarrow M_{2}^{-} \rightarrow V_{3} \\
& \rightarrow M_{2}^{+} \rightarrow V_{2} \rightarrow M_{1}^{+} \rightarrow V_{1} \\
V_{1} & \rightarrow M_{1}^{-} \rightarrow V_{2} \rightarrow M_{2}^{-} \rightarrow V_{3} \\
& \rightarrow M_{2}^{+} \rightarrow V_{2} \rightarrow M_{2}^{-} \\
& \rightarrow \cdots \rightarrow V_{2} \rightarrow M_{1}^{+} \rightarrow V_{1} .
\end{aligned}
$$

In the following, a 2D Poincarée return map is rigorously constructed for verifying the chaotic behaviors of the double-scroll attractor.

Without loss of generality, consider a trajectory started from an initial point $\left(1, y_{0}, z_{0}\right) \in M_{1}$ at $t=0$. If $\left(1, y_{0}, z_{0}\right) \in M_{1}-$ $M_{1}^{-}$, according to the exact solution (33), it must reach $M_{1}^{-}$at the positive time $t_{1}$. Let $\left(1, y_{1}, z_{1}\right) \in M_{1}^{-}$be the hitting point. One can get the arriving time $t_{1}$ and the positions $y_{1}$ and $z_{1}$ by using the solution (33). If $\left(1, y_{0}, z_{0}\right) \in M_{1}^{-}$, according to the solution (34), it must reach $M_{1}$ and $M_{2}$ at the positive time $t_{2}$ and $t_{3}$, respectively, without switchings. Here, $t_{2}$ and $t_{3}$ are the first arriving time. Let $\left(1, y_{2}, z_{2}\right) \in M_{1}$ be the hitting point. One can get the arriving time $t_{2}$ and positions $y_{2}$ and $z_{2}$ by using the solution (34). Similarly, for the hitting point $\left(1, y_{3}, z_{3}\right) \in M_{2}$, one can get the arriving time $t_{3}$ and positions $y_{3}$ and $z_{3}$. Now, one can define a region by

$$
\Gamma=\left\{(1, y, z) \in M_{1}^{-} \mid t_{2}<t_{3}\right\} .
$$

Thus, if $\left(1, y_{0}, z_{0}\right) \in \Gamma$, this trajectory will arrive at $\left(1, y_{2}, z_{2}\right) \in$ $M_{1}^{+}$at the positive time $t_{2}$; if $\left(1, y_{0}, z_{0}\right) \in M_{1}^{-}-\Gamma$, this trajectory will reach $\left(-1, y_{3}, z_{3}\right) \in M_{2}^{-}$at the positive time $t_{3}$. According to the switching rules, this trajectory will go through switching plane $M_{2}^{-}$at time $t_{3}$ from $V_{2}$ to $V_{3}$. Due to the symmetry of the vector fields in both $V_{1}$ and $V_{3}$, a trajectory started from $\left(-1, y_{3}, z_{3}\right)$ in $V_{3}$ is symmetric to that started from $\left(1,-y_{3},-z_{3}\right)$ on $M_{1}^{+}$in $V_{1}$. Here, one can define a $2 \mathrm{D}$ Poincaré return map by

$$
\mathbf{F}: M_{1} \rightarrow M_{1},\left(y_{0}, z_{0}\right) \rightarrow\left\{\begin{array}{l}
\left(y_{1}, z_{1}\right) \text { for }\left(y_{0}, z_{0}\right) \in M_{1}-M_{1}^{-} \\
\left(y_{2}, z_{2}\right) \text { for }\left(y_{0}, z_{0}\right) \in \Gamma \\
\left(-y_{3},-z_{3}\right) \text { for }\left(y_{0}, z_{0}\right) \in M_{1}-\Gamma
\end{array}\right.
$$

$$
\begin{aligned}
& A_{1}^{\prime}=\frac{\left(\alpha^{\prime 2}+\beta^{\prime 2}\right) x(0)-2 \alpha^{\prime} y(0)+z(0)}{\left(\lambda_{1}^{\prime}-\alpha^{\prime}\right)^{2}+\beta^{\prime 2}} \\
& A_{2}^{\prime}=\frac{\left(\lambda_{1}^{\prime 2}-2 \alpha^{\prime} \lambda_{1}^{\prime}\right) x(0)+2 \alpha^{\prime} y(0)-z(0)}{\left(\lambda_{1}^{\prime}-\alpha^{\prime}\right)^{2}+\beta^{\prime 2}} \\
& A_{3}^{\prime}=\frac{\left(\lambda_{1}^{\prime} \alpha^{\prime 2}-\lambda_{1}^{\prime} \beta^{\prime 2}-\lambda_{1}^{\prime 2} \alpha^{\prime}\right) x(0)-\left(\beta^{\prime 2}-\alpha^{\prime 2}+\lambda_{1}^{\prime 2}\right) y(0)+\left(\alpha^{\prime}-\lambda_{1}^{\prime}\right) z(0)}{\beta^{\prime}\left[\left(\lambda_{1}^{\prime}-\alpha^{\prime}\right)^{2}+\beta^{\prime 2}\right]}
\end{aligned}
$$


Note that one can write a rigorously mathematical formulation for this map by using the exact solutions (33) and (34), as follows:

$$
\begin{aligned}
\left(\begin{array}{c}
1-10 \\
y_{1} \\
z_{1}
\end{array}\right) & =\left(\begin{array}{c}
g_{1}\left(t_{1}\right) \\
g_{2}\left(t_{1}\right) \\
g_{3}\left(t_{1}\right)
\end{array}\right)\left(\begin{array}{c}
1-10 \\
y_{0} \\
z_{0}
\end{array}\right) \\
\left(\begin{array}{c}
1 \\
y_{2} \\
z_{2}
\end{array}\right) & =\left(\begin{array}{l}
g_{1}^{\prime}\left(t_{2}\right) \\
g_{2}^{\prime}\left(t_{2}\right) \\
g_{3}^{\prime}\left(t_{2}\right)
\end{array}\right)\left(\begin{array}{c}
1 \\
y_{0} \\
z_{0}
\end{array}\right)
\end{aligned}
$$

and

$$
\left(\begin{array}{c}
-1 \\
y_{3} \\
z_{3}
\end{array}\right)=\left(\begin{array}{c}
g_{1}^{\prime}\left(t_{3}\right) \\
g_{2}^{\prime}\left(t_{3}\right) \\
g_{3}^{\prime}\left(t_{3}\right)
\end{array}\right)\left(\begin{array}{c}
1 \\
y_{0} \\
z_{0}
\end{array}\right)
$$

Moreover, the Jacobian of this map $\mathbf{F}$ is described by

$$
\mathrm{DF}= \begin{cases}\left(\begin{array}{ll}
\frac{\partial y_{1}}{\partial y_{0}} & \frac{\partial y_{1}}{\partial z_{0}} \\
\frac{\partial z_{1}}{\partial y_{0}} & \frac{\partial z_{1}}{\partial z_{0}}
\end{array}\right), & \text { for }\left(y_{0}, z_{0}\right) \in M_{1}-M_{1}^{-} \\
\left(\begin{array}{ll}
\frac{\partial y_{2}}{\partial y_{0}} & \frac{\partial y_{2}}{\partial z_{0}} \\
\frac{\partial z_{2}}{\partial y_{0}} & \frac{\partial z_{2}}{\partial z_{0}}
\end{array}\right), & \text { for }\left(y_{0}, z_{0}\right) \in \Gamma \\
-\left(\begin{array}{ll}
\frac{\partial y_{3}}{\partial y_{0}} & \frac{\partial y_{3}}{\partial z_{0}} \\
\frac{\partial z_{3}}{\partial y_{0}} & \frac{\partial z_{3}}{\partial z_{0}}
\end{array}\right), & \text { for }\left(y_{0}, z_{0}\right) \in M_{1}-\Gamma .\end{cases}
$$

Therefore, one can get the exact mathematical formulations for the Lyapunov exponents $\mathrm{LE}_{1}, \mathrm{LE}_{2}$ of the mapping $\mathbf{F}$ from (36). However, the formulations are rather complex. In real calculations, one can use the numerical methods described in [29] to calculate the maximum Lyapunov exponent $\mathrm{LE}_{1}$. If $0<$ $\mathrm{LE}_{1}<+\infty$, system (11) has a double-scroll chaotic attractor. Fig. 10(a) shows the Poincaré mapping of double-scroll attractor at section $M_{1}$; Fig. 10(b) displays the Poincaré mapping of the double-scroll attractor at section $M_{2}$. It is clear that these two mappings are symmetric from a theoretical view, but there are some small differences due to calculating errors. Moreover, one can also rigorously prove the chaotic behavior of the doublescroll attractor by using the Homoclinic Šilnikov Theorem in [19]. The detailed proof is similar to the proof of Chua's doublescroll attractor [18] and thus is omitted here.

\section{B. Dynamical Analysis of Multi-Scroll Systems}

Here, the dynamical behaviors of multiscroll systems (12), (17), and (24) are briefly discussed.

Note that the multiscroll systems (12), (17), and (24) can be represented by a unified hybrid system, which can be regarded as a linear system with a saturated function series PWL feedback controller. It is described by

$$
\dot{\mathbf{X}}=\mathbf{A X}+\mathbf{B} \varphi(\mathbf{C X})
$$

where $\mathbf{X}=(x, y, z)^{T}$ is the state vector, and

$$
\begin{aligned}
\mathbf{A} & =\left(\begin{array}{ccc}
0 & 1 & 0 \\
0 & 0 & 1 \\
-a & -b & -c
\end{array}\right) \\
\mathbf{B} & =\left(\begin{array}{ccc}
0 & -\frac{d_{2}}{b} & 0 \\
0 & 0 & -\frac{d_{3}}{c} \\
d_{1} & d_{2} & d_{3}
\end{array}\right) \\
\mathbf{C} & =\left(\begin{array}{lll}
1 & 0 & 0 \\
0 & 1 & 0 \\
0 & 0 & 1
\end{array}\right) .
\end{aligned}
$$

Clearly, it can be classified into three different cases as follows: a) 1-D $n$-scroll chaotic attractor: With

$$
\varphi(\mathbf{C X})=\left(\begin{array}{c}
f\left(x ; k_{1}, h_{1}, p_{1}, q_{1}\right) \\
0 \\
0
\end{array}\right)
$$

system (37) becomes system (12).

b) 2-D $n \times m$-grid scroll chaotic attractor: With

$$
\varphi(\mathbf{C X})=\left(\begin{array}{c}
f\left(x ; k_{1}, h_{1}, p_{1}, q_{1}\right) \\
f\left(y ; k_{2}, h_{2}, p_{2}, q_{2}\right) \\
0
\end{array}\right)
$$

system (37) becomes system (17).

c) 3-D $n \times m \times l$-grid scroll chaotic attractor: With

$$
\varphi(\mathbf{C X})=\left(\begin{array}{l}
f\left(x ; k_{1}, h_{1}, p_{1}, q_{1}\right) \\
f\left(y ; k_{2}, h_{2}, p_{2}, q_{2}\right) \\
f\left(z ; k_{3}, h_{3}, p_{3}, q_{3}\right)
\end{array}\right)
$$

system (37) becomes system (24).

Suykens et al. discussed the similar link between the multiscroll chaotic attractors and the recurrent neural networks in [4]. It should be pointed out that system (37) has some very good analytical properties. This is because system (37) is continuous in $\mathbf{R}^{3}$. However, the corresponding hysteresis multiscroll system in [16] and stair multiscroll systems in [10] are discontinuous in all switching points.

Assume that $a=d_{1}, b=d_{2}, c=d_{3}, h_{1}=2 k_{1}, h_{2}=$ $2 k_{2}, h_{3}=2 k_{3}$. Consider system (12). When $x \in\left[i h_{1}-\right.$ $\left.\left(h_{1} / 2\right), i h_{1}+\left(h_{1} / 2\right)\right]\left(p_{1}-1 \leq i \leq q_{1}-1\right)$, let $x=x^{\prime}+i h_{1}, y=$ $y^{\prime}, z=z^{\prime}$. Then, $f\left(x ; k_{1}, h_{1}, p_{1}, q_{1}\right)=f_{0}\left(x^{\prime} ; k_{1}\right)+2 k_{1} i$ from (5). Therefore, system (12) becomes

$$
\left\{\begin{array}{l}
\dot{x^{\prime}}=y^{\prime} \\
\dot{y^{\prime}}=z^{\prime} \\
\dot{z}^{\prime}=-a x^{\prime}-b y^{\prime}-c z^{\prime}+d_{1} f_{0}\left(x^{\prime} ; k_{1}\right)
\end{array}\right.
$$

where $x^{\prime} \in\left[-\left(h_{1} / 2\right),\left(h_{1} / 2\right)\right]$.

It is noticed that this system is the double-scroll system (11) with $x \in\left[-\left(h_{1} / 2\right),\left(h_{1} / 2\right)\right]$. When $x \geq q_{1} h_{1}-\left(h_{1} / 2\right)$ (or $x \leq-p_{1} h_{1}+\left(h_{1} / 2\right)$ ), let $x=x^{\prime}+q_{1} h_{1}, \bar{y}=y^{\prime}, z=z^{\prime}$ (or $x=$ $\left.x^{\prime}-p_{1} h_{1}, y=y^{\prime}, z=z^{\prime}\right)$. Then, system (17) is equivalent to the double-scroll system (11) with $x^{\prime} \geq-\left(h_{1} / 2\right)$ (or $x \leq\left(h_{1} / 2\right)$ ). Similarly, for the multiscroll system (17), let $x=x^{\prime}+i h_{1}, y=$ $y^{\prime}+j h_{2}, z=z^{\prime}$. Then, system (17) becomes the double-scroll system (11) in the corresponding subspaces. For the multiscroll system (24), let $x=x^{\prime}+i h_{1}, y=y^{\prime}+j h_{2}, z=z^{\prime}+k h_{3}$. Then system (24) is equivalent to the double-scroll system (11) in the corresponding subspaces. That is, in each subspace, system (37) has the same dynamical behaviors as the double-scroll system (11). In essence, system (37) assembles the dynamical behaviors of system (11) with different initial values in different subspaces by its displacements in $x, y, z$-directions. It is easy to understand why the saturated plateaus of system (37) play an important role in the process of multiscroll generation.

According to (5), parameters $p_{i}, q_{i}(1 \leq i \leq 3)$ determine the number of the saturated plateaus in the $x, y, z$-directions. Moreover, each saturated plateau corresponds to a unique scroll and also determines the position of the scroll. In fact, one can arbitrarily design the number and also the position of the scrolls of the multiscroll system (37). One can also rotate the multiscroll chaotic attractors to any desired orientation in the state space. 


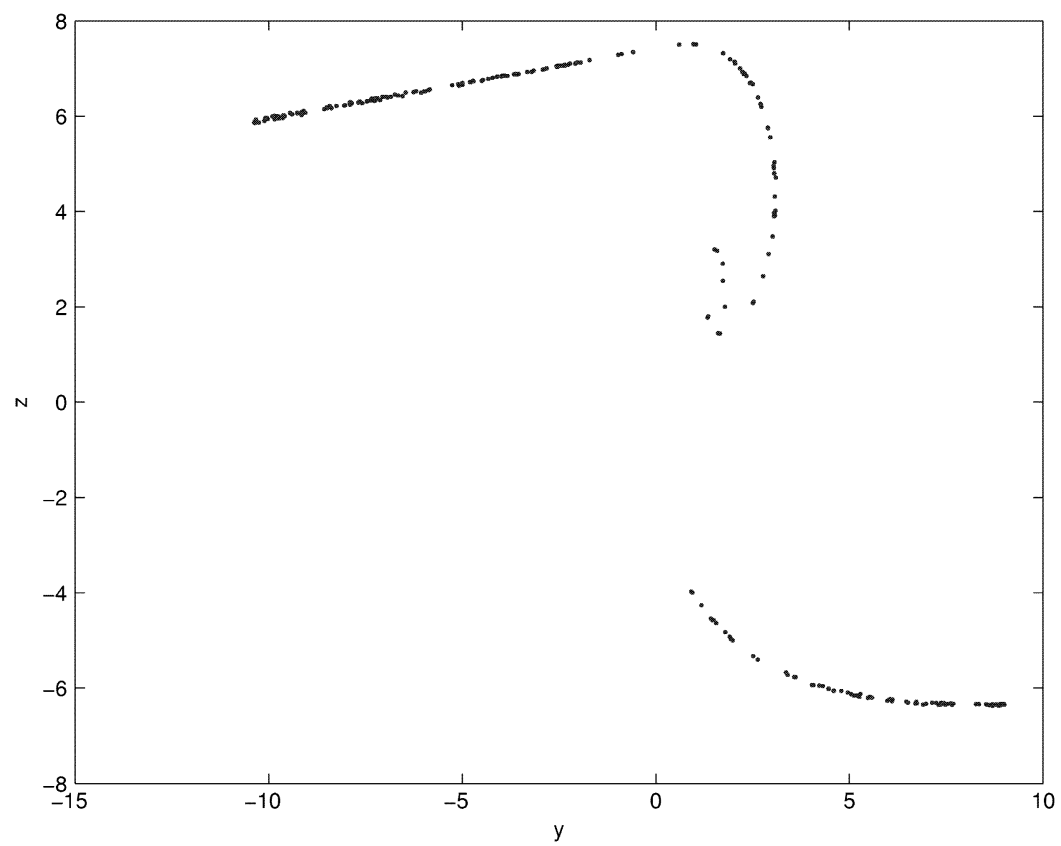

(a)

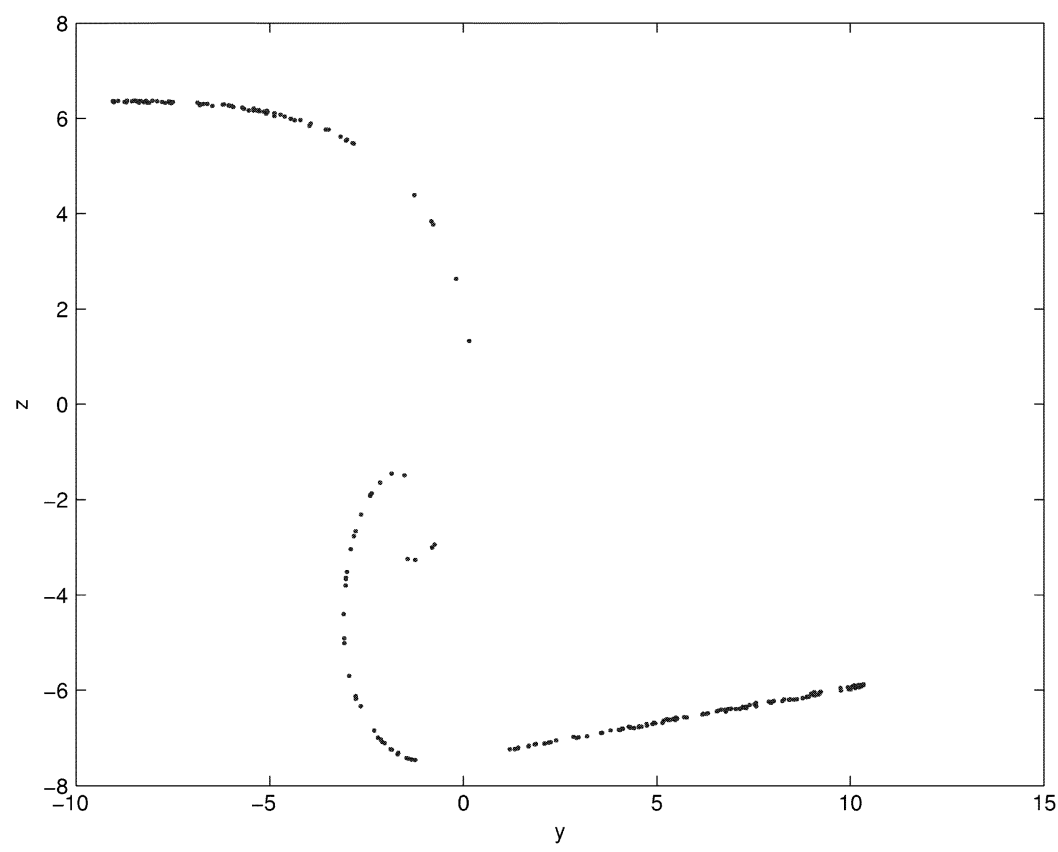

(b)

Fig. 10. Poincaré mapping of the double-scroll attractor. (a) Section $M_{1}$. (b) Section $M_{2}$.

Every PWL part has one and only one equilibrium point; however, only the equilibrium points in the saturated plateaus can generate scrolls. The trajectories of system (37) are repeatedly stretched and folded in the state space for infinitely many times via saturated function series switchings, leading to the appearance of complex dynamical behaviors. Note that system (37) is PWL and has an exact analytic solution in every subspace. However, the dynamical behaviors of the entire system become rather complex due to the saturated function series switchings.

Consider the 1-D $n$-scroll system (12). Obviously, system (12) has a 1-D $\left(p_{1}+q_{1}+2\right)$-scroll chaotic attractor, which has $2\left(p_{1}+\right.$ $\left.q_{1}\right)+3$ equilibria, located in the $x$-axis. Note that system (12) has $p_{1}+q_{1}+2$ saturated plateaus, and each saturated plateau corre- sponds to a unique equilibrium point of index 2 . Other $p_{1}+q_{1}+1$ equilibria of index 1 correspond to $p_{1}+q_{1}+1$ saturated slopes between these saturated plateaus, respectively. Denote

$$
\begin{aligned}
& E_{-p_{1}}=\left(-\infty,-p_{1} h_{1}+\frac{h_{1}}{2}\right] \\
& E_{i}= {\left[i h_{1}-\frac{h_{1}}{2}, i h_{1}+\frac{h_{1}}{2}\right], } \\
& \text { for }-p_{1}+1 \leq i \leq q_{1}-1 \\
& E_{q_{1}}=\left[q_{1} h_{1}-\frac{h_{1}}{2}+\infty\right) .
\end{aligned}
$$

When $h_{1}=2 k_{1}$, for each interval $E_{i}\left(-p_{1} \leq i \leq q_{1}\right)$ of variable $x$, system (12) has the same dynamical behaviors as the double- 
scroll system (11), which can be verified by using a displacement transformation $x^{\prime}=x-i h_{1}, y^{\prime}=y, z^{\prime}=z$ with $-p_{1} \leq i \leq q_{1}$. Thus, one can get the exact solution of system (12) in each subspace. When $p_{1}=q_{1}$, system (12) has a natural symmetry under the coordinates transform $(x, y, z) \rightarrow(-x,-y,-z)$, which persists for all values of the system parameters.

Consider the 2-D $n \times m$-grid scroll system (17). Clearly, system (17) has a 2 -D $\left(p_{1}+q_{1}+2\right) \times\left(p_{2}+q_{2}+2\right)$-grid scroll chaotic attractor, which has $\left(2 p_{1}+2 q_{1}+3\right) \times\left(2 p_{2}+2 q_{2}+3\right)$ equilibria, located in the $x-y$ plane. It is noticed that system (17) has $\left(p_{1}+q_{1}+2\right) \times\left(p_{2}+q_{2}+2\right) 2$-D saturated plateaus, and each saturated plateau corresponds to a unique equilibrium point of index 2. Other equilibria correspond to the saturated slopes between these saturated plateaus, respectively.

Denote

$$
\begin{aligned}
F_{-p_{2}} & =\left(-\infty,-p_{2} h_{2}+\frac{h_{2}}{2}\right] \\
F_{j} & =\left[j h_{2}-\frac{h_{2}}{2}, j h_{2}+\frac{h_{2}}{2}\right], \\
& \text { for }-p_{2}+1 \leq j \leq q_{2}-1 \\
F_{q_{2}} & =\left[q_{2} h_{2}-\frac{h_{2}}{2},+\infty\right) .
\end{aligned}
$$

When $h_{1}=2 k_{1}, h_{2}=2 k_{2}$, for each region $\Omega_{i j}=\{(x, y) \mid x \in$ $\left.E_{i}, y \in F_{j}\right\}\left(-p_{1} \leq i \leq q_{1} ;-p_{2} \leq j \leq q_{2}\right)$, system (17) has the same dynamical behaviors as the double-scroll system (11), which can be verified by using a displacement transformation $x^{\prime}=x-i h_{1}, y^{\prime}=y-j h_{2}, z^{\prime}=z$ for $-p_{1} \leq i \leq q_{1}$ and $-p_{2} \leq j \leq q_{2}$. Thus, one can get the exact solution of system (17) in each subspace. When $p_{1}=q_{1}, p_{2}=q_{2}$, system (17) has a natural symmetry under the coordinates transform $(x, y, z) \rightarrow(-x,-y,-z)$, which persists for all values of the system parameters.

Consider the 3-D $n \times m \times l$-grid scroll system (24). Obviously, system (24) has a $3-\mathrm{D}\left(p_{1}+q_{1}+2\right) \times\left(p_{2}+q_{2}+2\right) \times\left(p_{3}+q_{3}+\right.$ $2)$-grid scroll chaotic attractor, which has $\left(2 p_{1}+2 q_{1}+3\right) \times$ $\left(2 p_{2}+2 q_{2}+3\right) \times\left(2 p_{3}+2 q_{3}+3\right)$ equilibria. Notice that system (24) has $\left(p_{1}+q_{1}+2\right) \times\left(p_{2}+q_{2}+2\right) \times\left(p_{3}+q_{3}+2\right) 3$-D saturated plateaus, and each saturated plateau corresponds to a unique equilibrium point of index 2. Other equilibria correspond to the saturated slopes between these saturated plateaus, respectively.

Denote

$$
\begin{aligned}
G_{-p_{3}} & =\left(-\infty,-p_{3} h_{3}+\frac{h_{3}}{2}\right] \\
G_{k}= & {\left[k h_{3}-\frac{h_{3}}{2}, k h_{3}+\frac{h_{3}}{2}\right], } \\
G_{q_{3}} & =\left[q_{3} h_{3}-\frac{h_{3}}{2}+\infty\right) .
\end{aligned}
$$

When $h_{1}=2 k_{1}, h_{2}=2 k_{2}, h_{3}=2 k_{3}$, for each subspace $\Gamma_{i j k}=\left\{(x, y, z) \mid x \in E_{i}, y \in F_{j}, z \in G_{k}\right\}\left(-p_{1} \leq i \leq\right.$ $q_{1} ;-p_{2} \leq j \leq q_{2} ;-p_{3} \leq k \leq q_{3}$ ), system (24) has the same dynamical behaviors as the double-scroll system (11), which can be verified by using a displacement transformation $x^{\prime}=x-$ $i h_{1}, y^{\prime}=y-j h_{2}, z^{\prime}=z-k h_{3}$ for $-p_{1} \leq i \leq q_{1},-p_{2} \leq j \leq$ $q_{2}$, and $-p_{3} \leq k \leq q_{3}$. Then, one can get the exact solution of system (24) in each subspace. When $p_{1}=q_{1}, p_{2}=q_{2}, p_{3}=q_{3}$, system (24) has a natural symmetry under the coordinates transform $(x, y, z) \rightarrow(-x,-y,-z)$, which persists for all values of the system parameters.

Similarly, the 2D Poincaré return maps can be constructed and used to prove the chaotic behaviors of the multiscroll systems (12), (17), and (24). However, their constructing processes are similar but rather complex, and hence are omitted here.

\section{CONCLUSION}

This paper has initiated a saturated function series approach for generating multiscroll chaotic attractors, including 1-D $n$-scroll, 2-D $n \times m$-grid scroll, and 3-D $n \times m \times l$-grid scroll attractors, from a given 3D linear autonomous system with a saturated function series as the controller. The dynamical behaviors and chaos generation mechanism of multiscroll systems have been further investigated by analyzing the system trajectories. In particular, a 2D Poincaré return map has been rigorously constructed for verifying the chaotic behaviors of the double-scroll attractor.

It should be pointed out that one can arbitrarily design a desired number of scrolls and their spatial positions and orientations by using this developed systematic methodology. Furthermore, it is relatively easy to design physical electronic circuits to experimentally verify these multiscroll chaotic attractors since the saturated circuit is a basic electrical circuit.

Finally, it can be foreseen that various related bifurcation phenomena in the generated multiscroll chaotic systems deserve further investigation in the near future.

\section{REFERENCES}

[1] G. Chen and X. Yu, Eds., Chaos Control: Theory and Applications. Heidelberg, Germany: Springer-Verlag, 2003.

[2] G. Chen and J. Lü, Dynamics of the Lorenz System Family: Analysis, Control, and Synchronization, Beijing, China: Science Press, 2003.

[3] J. A. K. Suykens and J. Vandewalle, "Generation of $n$-double scrolls ( $n=1,2,3,4, \ldots)$,' IEEE Trans. Circuits Syst. I, vol. 40, pp. 861-867, Nov. 1993.

[4] J. A. K. Suykens, A. Huang, and L. O. Chua, "A family of $n$-scroll attractors from a generalized Chua's circuit," Int. J. Electron. Commun., vol. 51, no. 3, pp. 131-138, 1997.

[5] J. A. K. Suykens and L. O. Chua, " $n$-double scroll hypercubes in 1-D CNNs," Int. J. Bifurc. Chaos, vol. 7, no. 8, pp. 1873-1885, 1997.

[6] M. E. Yalcin, S. Ozoguz, J. A. K. Suykens, and J. Vandewalle, " $n$-scroll chaos generators: A simple circuit model," Electron. Lett., vol. 37, no. 3, pp. 147-148, 2001.

[7] P. Arena, S. Baglio, L. Fortuna, and G. Manganaro, "Generation of n-double scrolls via cellular neural networks," Int. J. Circuit Theory Applicat., vol. 24, no. 3, pp. 241-252, 1996.

[8] K. S. Tang, G. Q. Zhong, G. Chen, and K. F. Man, "Generation of $n$-scroll attractors via sine function," IEEE Trans. Circuits Syst. I, vol. 48, pp. 1369-1372, Nov. 2001.

[9] M. E. Yalcin, S. Ozoguz, J. A. K. Suykens, and J. Vandewalle, "Scroll maps from $n$-scroll attractors," in Proc. 10th Workshop on Nonlinear Dynamics of Electronic Systems, vol. 2, Izmir, Turkey, June 21-23, 2002, pp. $45-48$.

[10] M. E. Yalcin, J. A. K. Suykens, J. Vandewalle, and S. Ozoguz, "Families of scroll grid attractors," Int. J. Bifurc. Chaos, vol. 12, no. 1, pp. 23-41, Jan. 2002.

[11] S. Ozoguz, A. S. Elwakil, and K. N. Salama, " $n$-scroll chaos generator using nonlinear transconductor," Electron. Lett., vol. 38, pp. 685-686, 2002.

[12] G. Zhong, K. F. Man, and G. Chen, "A systematic approach to generating $n$-scroll attractors," Int. J. Bifurc. Chaos, vol. 12, no. 12, pp. 2907-2915, 2002.

[13] J. Lü, T. Zhou, G. Chen, and X. Yang, "Generating chaos with a switching piecewise-linear controller," Chaos, vol. 12, no. 2, pp. 344-349, 2002. 
[14] J. Lü, X. Yu, and G. Chen, "Generating chaotic attractors with multiple merged basins of attraction: A switching piecewise-linear control approach," IEEE Trans. Circuits Syst. I, vol. 50, pp. 198-207, Feb. 2003.

[15] F. Han, J. Lü, X. Yu, G. Chen, and Y. Feng, "Generating multiscroll chaotic attractors via a linear second-order hysteresis system," Dyn. Continuous, Discrete, Impulsive Syst., to be published.

[16] J. Lü, F. Han, X. Yu, and G. Chen, "Generating 3-D multiscroll chaotic attractors: A hysteresis series switching method," Automatica, vol. 40, no. 11, pp. 1677-1877, 2004.

[17] D. Cafagna and G. Grassi, "New 3-D-scroll attractors in hyperchaotic Chua's circuit forming a ring," Int. J. Bifur. Chaos, vol. 13, no. 10, pp. 2889-2903, 2003.

[18] L. O. Chua, M. Komuro, and T. Matsumoto, "The double scroll family," IEEE Trans. Circuits Syst., vol. 33, pp. 1072-1118, Nov. 1986.

[19] C. P. Silva, "Shil'nikov's theorem-A tutorial," IEEE Trans. Circuits Syst. I, vol. 40, pp. 675-682, Oct. 1993.

[20] A. S. Elwakil and M. P. Kennedy, "Construction of classes of circuitindependent chaotic oscillators using passive-only nonlinear devices," IEEE Trans. Circuits Syst. I, vol. 48, pp. 289-307, Mar. 2001.

[21] A. S. Elwakil, K. N. Salama, and M. P. Kennedy, "A system for chaos generation and its implementation in monolithic form," in Proc. IEEE Int. Symp. Circuits Systems, vol. 5, Geneva, Switzerland, 2000, pp. $217-220$

[22] T. Saito and S. Nakagawa, "Chaos from a hysteresis and switched circuit," Phil. Trans. R. Soc. Lond. A, vol. 353, pp. 47-57, 17011995.

[23] S. Nakagawa and T. Saito, "An $R C$ OTA hysteresis chaos generator," IEEE Trans. Circuits Syst. I, vol. 43, pp. 1019-1021, Dec. 1996.

[24] A. S. Kennedy and M. P. Kennedy, "Chaotic oscillators derived from Saito's double-screw hysteresis oscillator," IEICE Trans. Fund., vol. E82A, no. 9, pp. 1769-1775, 1999.

[25] A. S. Elwakil and M. P. Kennedy, "Systematic realization of a class of hysteresis chaotic oscillators," Int. J. Circuit Theory Applicat., vol. 28, pp. 319-334, 2000.

[26] T. Saito, "An approach toward higher dimensional hysteresis chaos generators," IEEE Trans. Circuits Syst. I, vol. 37, pp. 399-409, Mar. 1990.

[27] J. Lü, X. Yu, and G. Chen, "Switching control for multiscroll chaos generation: An overview," in Proc. Physics and Control, vol. 3, Saint Petersburg, , Russia, Aug. 20-22, 2003, pp. 420-428.

[28] J. Vandewalle and L. Vandenberghe, "Piecewise-linear circuits and piecewise-linear analysis," in The Circuits and Filters Handbook, W. K. Chen, Ed. Boca Raton, FL: CRC, 1995, pp. 1034-1057.

[29] J. Lü, J. Lu, and S. Chen, Chaotic Time Series Analysis and Its Applications. Wuhan, China: Wuhan Univ. Press, 2002.

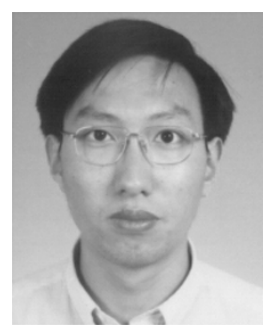

Jinhu Lü (M'03) received the B.Sc. degree in mathematics, the M.Sc. degree in applied mathematics, and the Ph.D. degree in applied mathematics, from Hubei Normal University, Hubei, China, Wuhan University, Wuhan, China, and the Chinese Academy of Sciences, Beijing, China, in 1997, 2000, and 2002, respectively.

From January to April of 2002, he was a Research Assistant, and from August to November of 2003, a Senior Research Associate at the Centre for Chaos Control and Synchronization, City University of Hong Kong, Hong Kong SAR, China. From January to May 2003, he was a Visiting Research Fellow in the School of Electrical and Computer Engineering, Royal Melbourne Institute of Technology, Melbourne, Australia. From June 2002 to February 2004, he was a Postdoctoral Research Fellow, and from March 2004, an Assistant Professor with the Institute of Systems Science, Academy of Mathematics and Systems Sciences, Chinese Academy of Sciences, Beijing, China. Currently, he is a Postdoctoral Fellow in the Department of Electrical and Computer Engineering, University of Calgary, Calgary, AB, Canada. He is the author of two research monographs and more than 50 research journal papers published in the fields of control and synchronization of complex dynamical systems.

Dr. Lü received the Presidential Outstanding Research Award from the Chinese Academy of Sciences in 2002 and the 100 National Best Ph.D. Theses Award from the Office of Academic Degrees Committee of the State Council and the Ministry of Education of China in 2004.

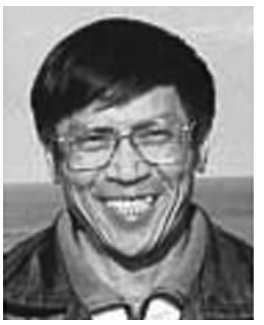

Guanrong Chen (M'87-SM'92-F'96) received the M.Sc. degree in computer science from the Sun Yat-sen (Zhongshan) University, China and the $\mathrm{Ph} . \mathrm{D}$. degree in applied mathematics from Texas A\&M University, College Station.

Currently he is a Chair Professor and the Founding Director of the Centre for Chaos Control and Synchronization at the City University of Hong Kong. He has (co)authored 15 research monographs and advanced textbooks, more than $300 \mathrm{SCI}$ journal papers, and about 200 refereed conference papers, published since 1981 in the fields of nonlinear system dynamics and controls. Among his publications are the research monographs entitled Hopf Bifurcation Analysis (Singapore: World Scientific, 1996), From Chaos to Order (Singapore: World Scientific, 1998), and edited books Controlling Chaos and Bifurcations in Engineering Systems (Boca Raton: FL, CRC, 1999), Chaos in Circuits and Systems (Singapore: World Scientific, 2002), Chaos Control, and Bifurcation Control (New York: Springer-Verlag, 2003).

Prof. Chen served and is serving as Chief Editor, Deputy Chief Editor, Advisory Editor, Features Editor, and Associate Editors for eight international journals including the IEEE TRANSACTIONS ON CIRCUITS AND SYSTEMS, IEEE TRANSACTIONS ON AUTOMATIC CONTROL, and the International Journal of Bifurcation and Chaos. He received the 1998 Harden-Simons Prize for the Outstanding Journal Paper Award from the American Society of Engineering Education and the $2001 \mathrm{M}$. Barry Carlton Best Annual Transactions Paper Award from the IEEE Aerospace and Electronic Systems Society. He is Honorary Professor of the Central Queensland University, Australia, as well as Honorary Guest-Chair Professor of more than ten Universities in China. He is a Fellow of the IEEE for his fundamental contributions to the theory and applications of chaos control and bifurcation analysis.

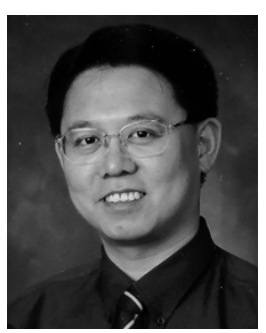

Xinghuo Yu (M'91-SM'96) received the B.E. and M.E. degrees from the University of Science and Technology of China, Hefei, China, in 1982 and 1985, respectively, and the Ph.D. degree from South-East University, Nanjing, China, in 1988.

Currently he is with the Royal Melbourne Institute of Technology (RMIT), Melbourne, Australia, where he is a full Professor and the Associate Dean Research \& Innovation of the Science, Engineering, and Technology Portfolio. He has held Visiting Professor positions in City University of Hong Kong and Bogazici University, Turkey. He is a Guest Chair Professor of four Chinese universities. His research interests include nonlinear and sliding mode control, intelligent control, intelligent systems and their industrial applications. He has published over 250 refereed papers in technical journals, books and conference proceedings, and also coedited seven research books.

Prof. Yu has served as an Associate Editor of IEEE TRANSACTIONS ON CIRCUITS AND SYSTEMS-I between 2001 and 2003, and is now serving as an Associate Editor of IEEE TRANSACTIONS ON INDUSTRIAL INFORMATICS. $\mathrm{He}$ is also on the editorial board of several other journals. He has been on the program committees of over 40 international and national conferences. He was the sole recipient of the 1995 Central Queensland University (CQU) Vice Chancellor's Award for Research. He is a Fellow of the Institution of Engineers Australia. He was made Professor Emeritus of CQU Australia in recognition of his significant contributions to the CQU.

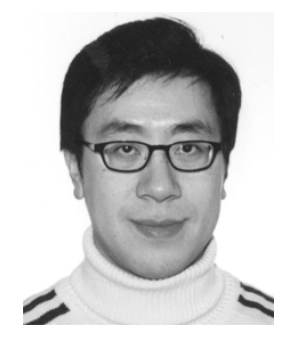

Henry Leung (M'90) received the Ph.D. degree in electrical and computer engineering from the McMaster University, Hamilton, ON, Canada.

$\mathrm{He}$ is currently a Professor in the Department of Electrical and Computer Engineering, University of Calgary, Calgary, AB, Canada. Prior to this, he was with the Defence Research Establishment, Ottawa, ON, Canada, where he was involved in the design of automated systems for air and maritime multisensor surveillance. His research interests include chaos, computational intelligence, data mining, nonlinear signal processing, multimedia, radar, sensor fusion, and wireless communications. 